Network Working Group

Request for Comments: 2677

Category: Standards Track
M. Greene

Contractor

J. Cucchiara

IronBridge Networks

J. Luciani

Bay Networks

August 1999

\title{
Definitions of Managed Objects for \\ the NBMA Next Hop Resolution Protocol (NHRP)
}

Status of this Memo

This document specifies an Internet standards track protocol for the Internet community, and requests discussion and suggestions for improvements. Please refer to the current edition of the "Internet Official Protocol standards" (STD 1) for the standardization state and status of this protocol. Distribution of this memo is unlimited.

Copyright Notice

Copyright (C) The Internet Society (1999). All Rights Reserved.

Abstract

This memo defines a portion of the Management Information Base (MIB) for use with network management protocols in the Internet community. In particular, it describes managed objects for the Next Hop Resolution Protocol (NHRP) as defined in RFC 2332.

Table of Contents

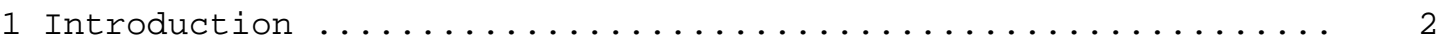

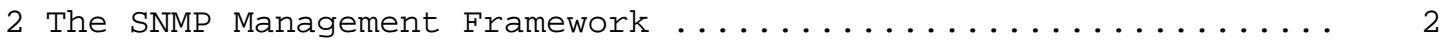

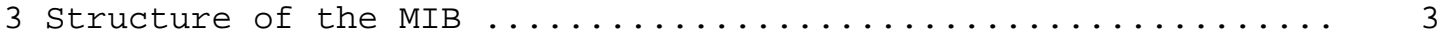

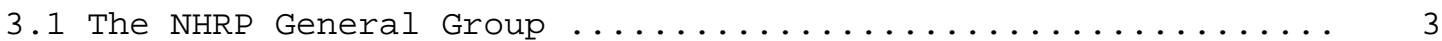

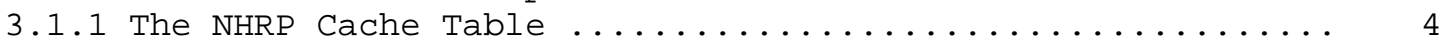

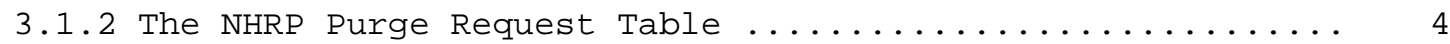

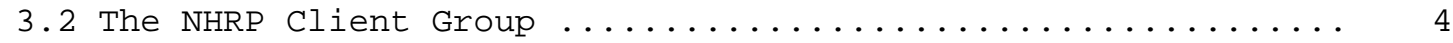

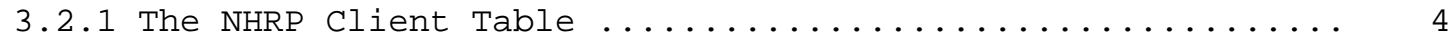

3.2.2 The NHRP Client Registration Table ................ 5

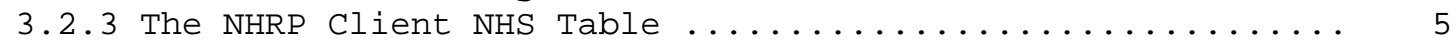

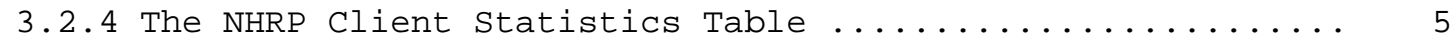

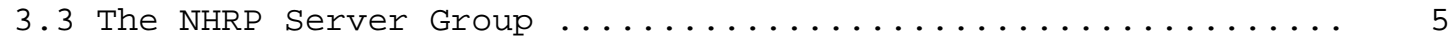

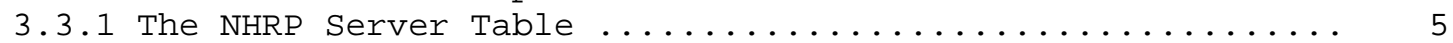

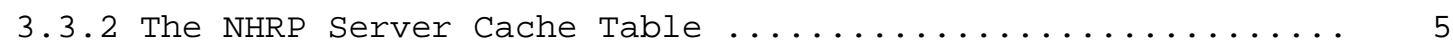

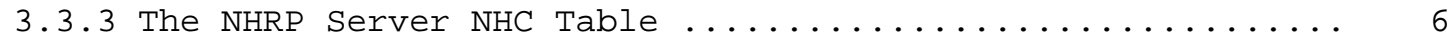




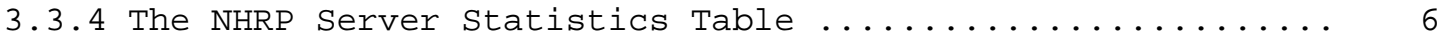

4 NBMA Next Hop Resolution Protocol MIB Definitions .......... 6

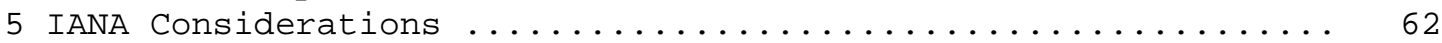

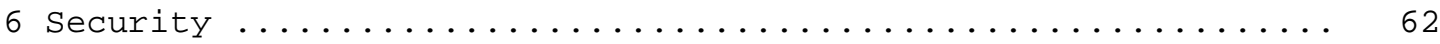

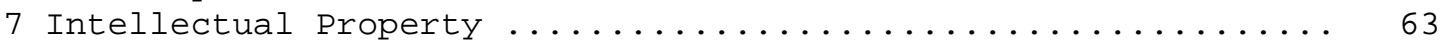

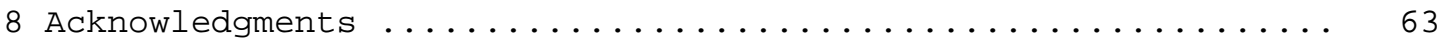

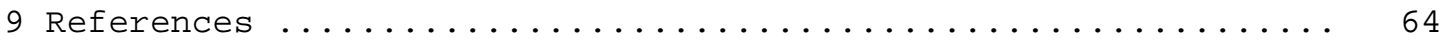

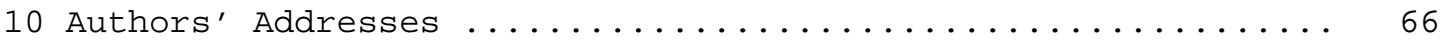

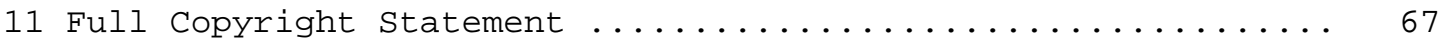

1. Introduction

This memo defines a portion of the Management Information Base (MIB) for use with network management protocols in the Internet community. In particular, it describes managed objects for the Next Hop Resolution Protocol (NHRP) as defined in RFC 2332 [17].

The key words "MUST", "MUST NOT", "REQUIRED", "SHALL", "SHALL NOT", "SHOULD", "SHOULD NOT", "RECOMMENDED", "MAY", and "OPTIONAL" in this document are to be interpreted as described in RFC 2119 [21].

\section{The SNMP Management Framework}

The SNMP Management Framework presently consists of five major components:

- An overall architecture, described in RFC 2571 [1].

- Mechanisms for describing and naming objects and events for the purpose of management. The first version of this structure of Management Information (SMI) is called SMIV1 and described in $\mathrm{STD} 16, \mathrm{RFC} 1155$ [2], STD 16, RFC 1212 [3] and RFC 1215 [4]. The second version, called SMIv2, is described in STD 58, RFC 2578 [5], STD 58, RFC 2579 [6] and STD 58, RFC 2580 [7].

- Message protocols for transferring management information. The first version of the SNMP message protocol is called SNMPv1 and described in STD 15, RFC 1157 [8]. A second version of the SNMP message protocol, which is not an Internet standards track protocol, is called SNMPv2c and described in RFC 1901 [9] and RFC 1906 [10]. The third version of the message protocol is called SNMPv3 and described in RFC 1906 [10], RFC 2572 [11] and RFC 2574 [12]. 
Protocol operations for accessing management information. The first set of protocol operations and associated PDU formats is described in STD 15, RFC 1157 [8]. A second set of protocol operations and associated PDU formats is described in RFC 1905 [13].

- A set of fundamental applications described in RFC 2573 [14] and the view-based access control mechanism described in RFC 2575 [15].

A more detailed introduction to the current SNMP Management Framework can be found in RFC 2570 [16].

Managed objects are accessed via a virtual information store, termed the Management Information Base or MIB. Objects in the MIB are defined using the mechanisms defined in the SMI.

This memo specifies a MIB module that is compliant to the SMIV2. A MIB conforming to the SMIv1 can be produced through the appropriate translations. The resulting translated MIB must be semantically equivalent, except where objects or events are omitted because no translation is possible (use of Counter64). Some machine readable information in SMIV2 will be converted into textual descriptions in SMIv1 during the translation process. However, this loss of machine readable information is not considered to change the semantics of the MIB.

3. Structure of the MIB

The NHRP MIB contains three groups: the General Group, the Client Group, and the Server Group.

\subsection{The NHRP General Group}

The General Group contains objects that apply to both clients and servers -- in particular the nhrpNextIndex scalar object, the NHRP Cache Table and the NHRP Purge Request Table.

The nhrpNextIndex scalar object is used to provide unique indices for the nhprclientIndex in the nhrpclientTable and the nhrpserverIndex in the nhrpserverTable. If used consistently, this object may prevent conflicts when multiple managers attempt to create rows simultaneously in the same table. 


\subsubsection{The NHRP Cache Table}

The NHRP Cache Table represents the internetwork layer address to NBMA address cache that is maintained by both NHRP clients and NHRP servers.

The NHRP Cache Table contains an ifIndex as part of the Index Clause. This ifIndex represents the use of a generic ifIndex, such that the value of this ifIndex SHOULD reflect a specific NBMA subnetwork related interface as determined by an implementation. For example, assuming that the NBMA subnetwork is ATM, then it is up to the implementors of this MIB to determine their own ATM interface layering (assuming compliance with the IF-MIB, RFC 2233 [18] and the ATM-MIB, RFC 2515 [19]). In other words, assuming that the NBMA subnetwork is ATM, the ifIndex in the NHRP Cache Table would represent the ifIndex containing or consisting of the VC (or shortcut) denoted by this Table entry.

The indexing scheme for the NHRP Cache Table is very similar to the MPC Ingress Cache Table and the MPS Ingress Cache Table in the

Multiprotocol Over ATM (MPOA) MIB [23]. This MIB and the MPOA MIB were designed to be complementary and non-overlapping. The MPOA MIB should also support this MIB. The MPOA MIB was designed prior to this MIB, and was designed by the LANE/MPOA Working Group in the ATM FORUM. The indexing scheme of the NHRP Cache Table (and the NHRP Server Cache Table) reflect the indexing scheme of the MPC Ingress Cache Table and the MPS Ingress Cache Table. Although, other indexing schemes could have been used for the NHRP Cache Table, a consistent indexing scheme between these tables was thought to be more advantageous from an implementation standpoint.

\subsubsection{The NHRP Purge Request Table}

The NHRP Purge Request Table is a way to track Purge Request Information.

\subsection{The NHRP Client Group}

The Client Group contains objects that only apply to NHRP clients (NHCs).

\subsubsection{The NHRP Client Table}

The NHRP Client Table contains entries for NHRP Next Hop Clients (NHCs) associated with this agent. Each row in the table represents a single NHC. The RequestID used in Registration requests needs to be saved to non-volatile storage. Depending upon the implementation, 
this may or may not impact how the storageType is used. For a complete description of how the Registration Requestid is used, see Section 5.2.3 of [17].

\subsubsection{The NHRP Client Registration Table}

The NHRP Client Registration Table contains information on registration requests which need to be maintained by the clients. Each entry in this table represents a single registration request. Note: since the NHRP specification does not mandate a refresh algorithm, this table omits refresh information, however, this table does contain information for all the registration requests which need to be maintained by the NHRP Clients.

3.2.3. The NHRP Client NHS Table

The NHRP Client NHS Table contains the NBMA subnetwork addresses of servers configured for use by the client. By default, the agent will add an entry to this table which corresponds to the client's default router.

\subsubsection{The NHRP Client Statistics Table}

The NHRP Client Statistics Table contains NHRP statistics maintained by a client. These statistics include counters on requests and replies, as well as counters for errors which are encountered by the clients.

\subsection{The NHRP Server Group}

The Server Group contains objects that only apply to NHRP servers (NHSes).

\subsubsection{The NHRP Server Table}

The NHRP Server Table contains entries for each server associated with this agent.

\subsubsection{The NHRP Server Cache Table}

The NHRP Server Cache Table contains additional objects that a server keeps for each entry in its cache. This table extends the NHRP Cache Table defined in the General Group. 
3.3.3. The NHRP Server NHC Table

This table contains information about all the clients known to the Servers.

3.3.4. The NHRP Server Statistics Table

The NHRP Server statistics Table contains NHRP statistics maintained by a server. These statistics include counters on requests and replies, as well as counters for errors which are encountered by the Servers.

4. NBMA Next Hop Resolution Protocol MIB Definitions

NHRP-MIB DEFINITIONS : := BEGIN

IMPORTS

OBJECT-TYPE, MODULE-IDENTITY, mib-2, Integer32,

Counter32, Unsigned32

FROM SNMPV2-SMI

MODULE-COMPLIANCE, OBJECT-GROUP

FROM SNMPV2-CONF

TEXTUAL-CONVENTION, TruthValue, RowStatus, StorageType,

Timestamp

FROM SNMPV2-TC

if Index

FROM IF-MIB

AddressFamilyNumbers

FROM IANA-ADDRESS-FAMILY-NUMBERS-MIB

;

nhrPMIB MODULE-IDENTITY

LAST-UPDATED "9908260000Z" -- August 26, 1999

ORGANIZATION "Internetworking Over NBMA (ion) Working Group" CONTACT-INFO

"Maria Greene (mariaexedia.com)

Contractor

Joan Cucchiara (joandironbridgenetworks.com)

IronBridge Networks

James V. Luciani (luciani@baynetworks.com)

Bay Networks" 


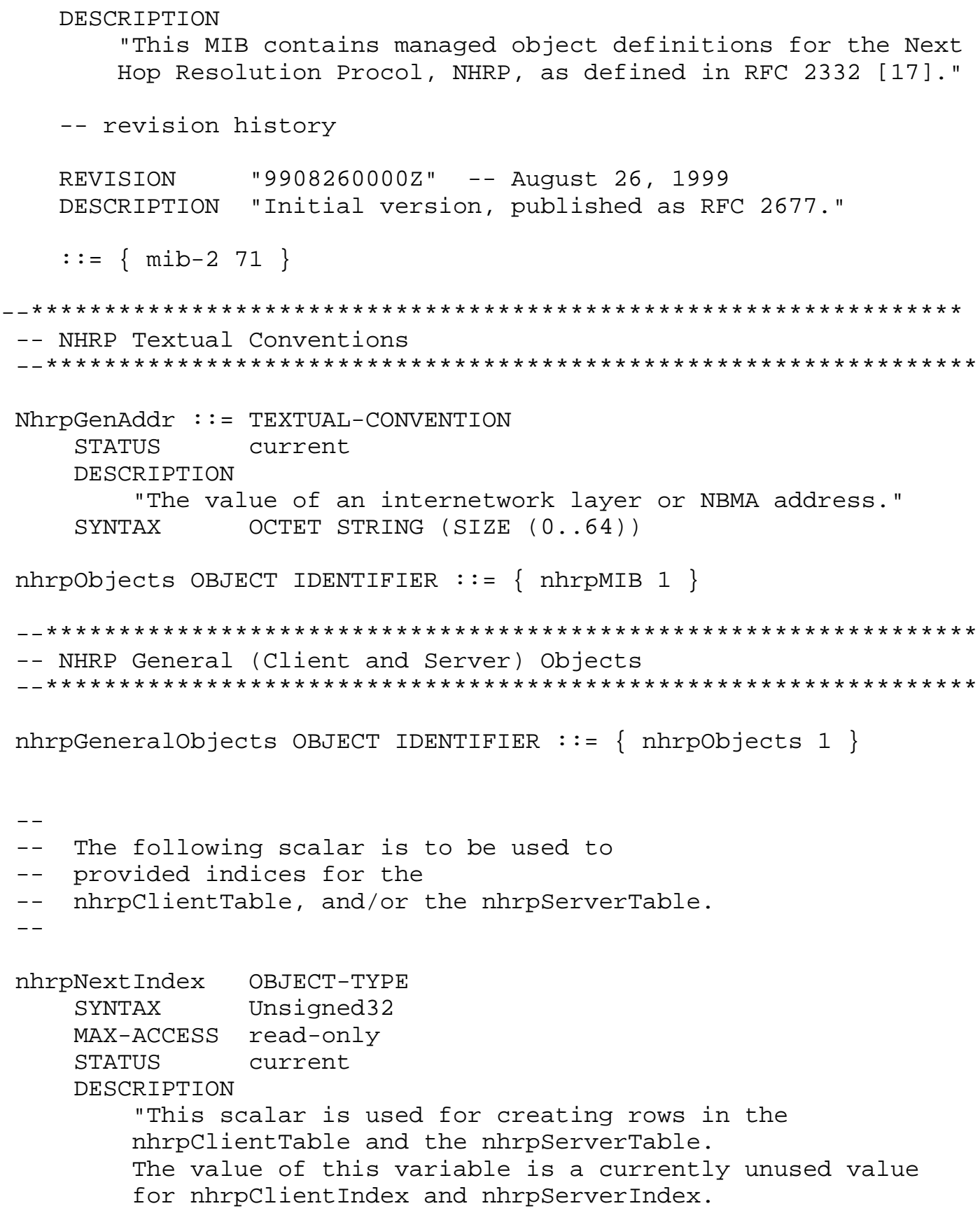


The value returned when reading this variable must be unique for the NHC's and NHS's indices associated with this row. Subsequent attempts to read this variable must return different values.

NOTE: this object exists in the General Group because it is to be used in establishing rows in the nhrpclientTable and the nhrpserverTable. In other words, the value retrieved from this object could become the value of nhrpclientIndex and nhprServerIndex.

In the situation of an agent re-initialization the value of this object must be saved in non-volatile storage.

This variable will return the special value 0 if no new rows can be created."

$::=\{$ nhrpGeneralobjects 1$\}$

$-1$

-- The NHRP Cache Table

$--$

nhrpCacheTable OBJECT-TYPE

SYNTAX SEQUENCE OF NhrpCacheEntry

MAX-ACCESS not-accessible

STATUS current

DESCRIPTION

"This table contains mappings between internetwork layer addresses and NBMA subnetwork layer addresses."

$::=\{$ nhrpGeneralobjects 2$\}$

nhrpCacheEntry OBJECT-TYPE

SYNTAX NhrpCacheEntry

MAX-ACCESS not-accessible

STATUS current

DESCRIPTION

"A cached mapping between an internetwork layer address and an NBMA address. Entries can be created by the network administrator using the nhrpcacheRowstatus column, or they may be added dynamically based on protocol operation (including NHRP, SCSP, and others, such as ATMARP).

When created based by NHRP protocol operations this entry is largely based on contents contained in the Client Information Entry (CIE). 


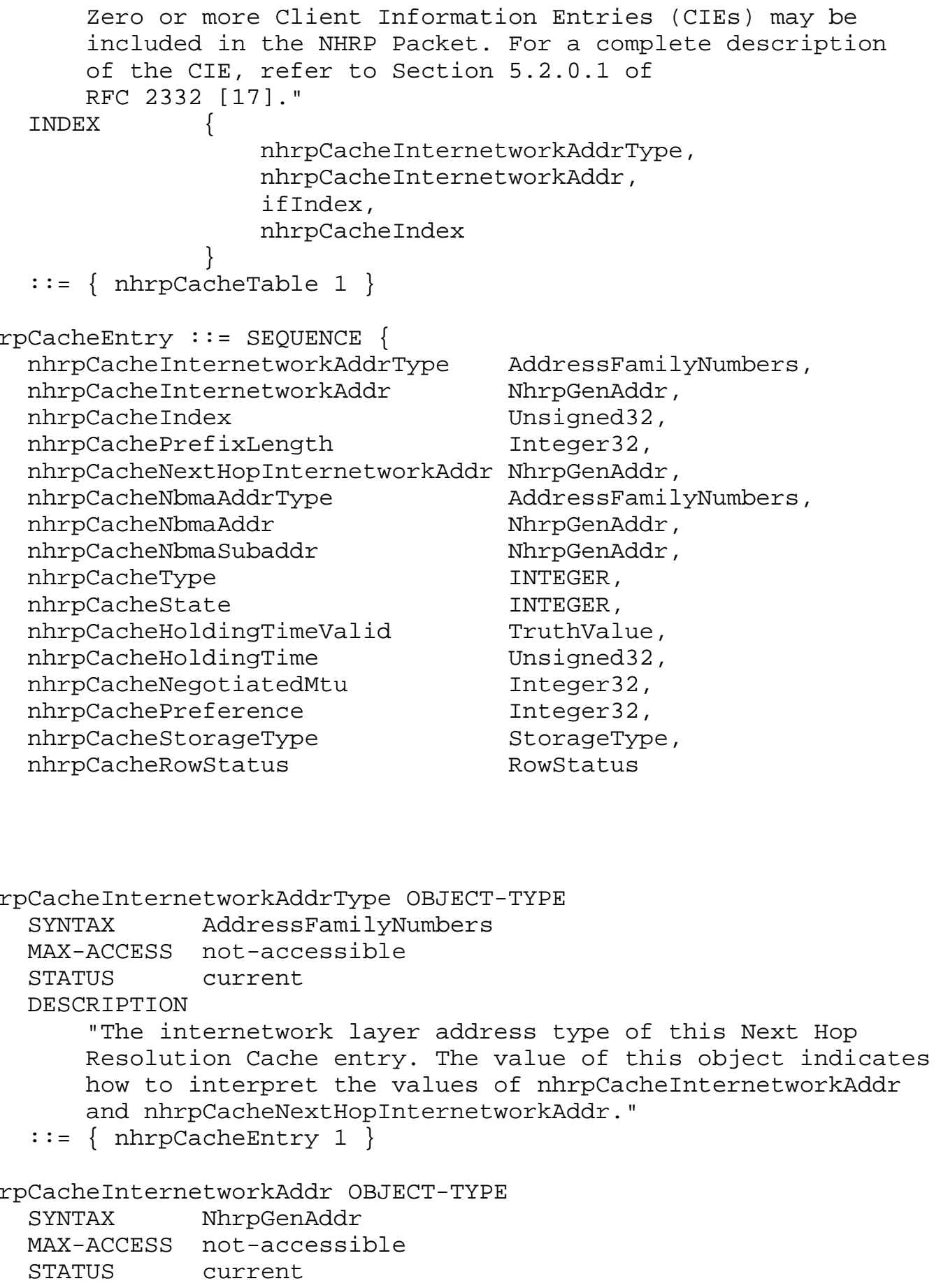




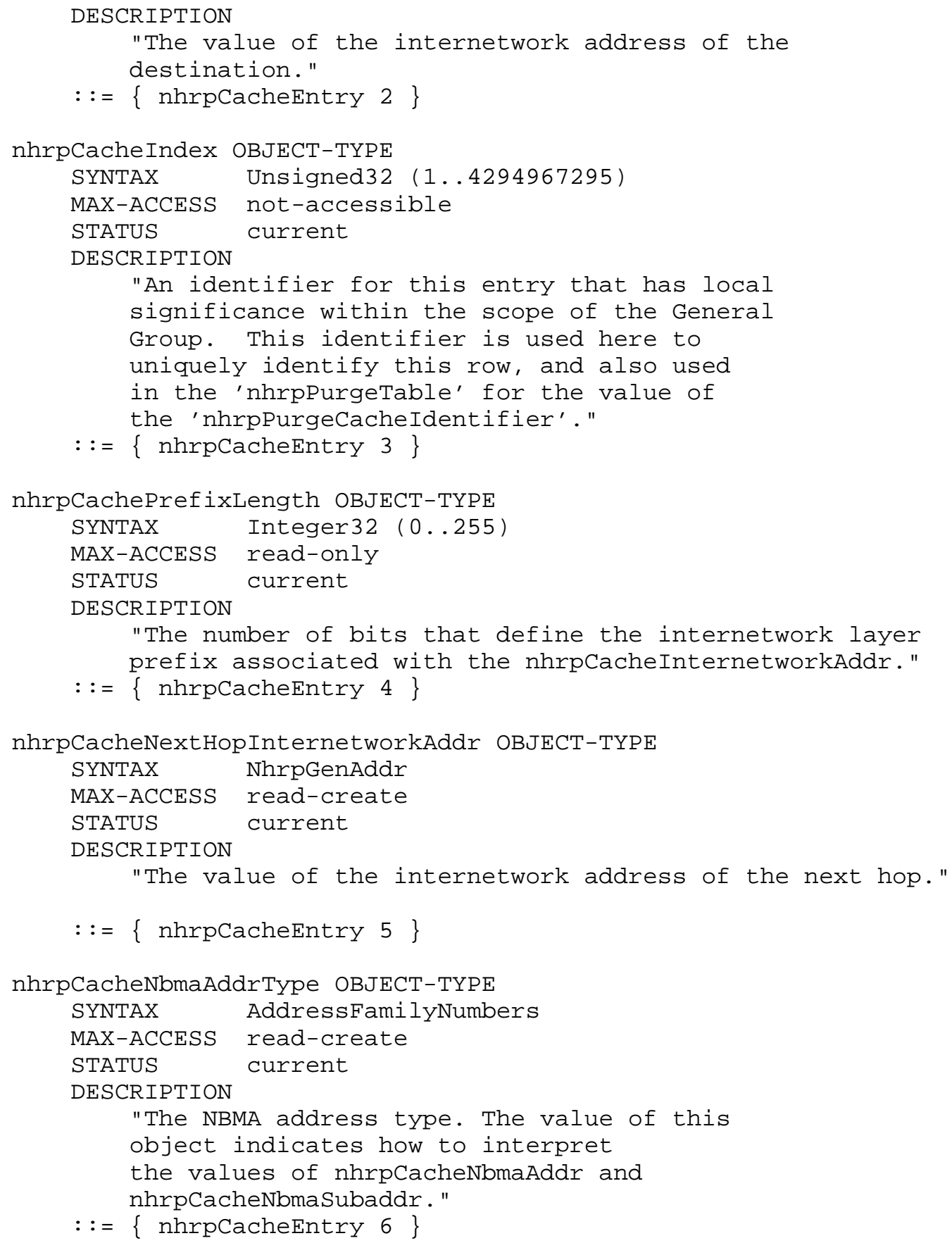




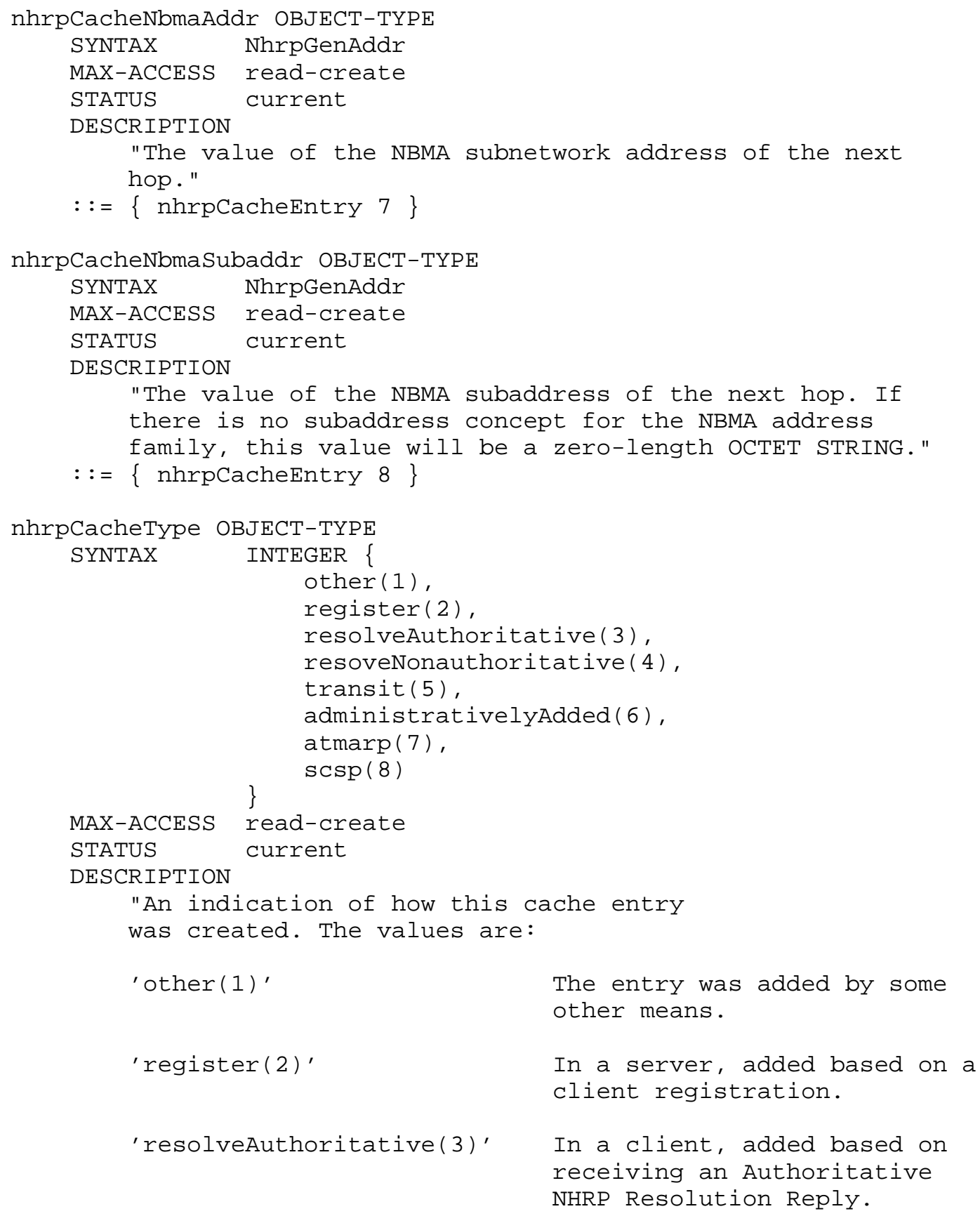




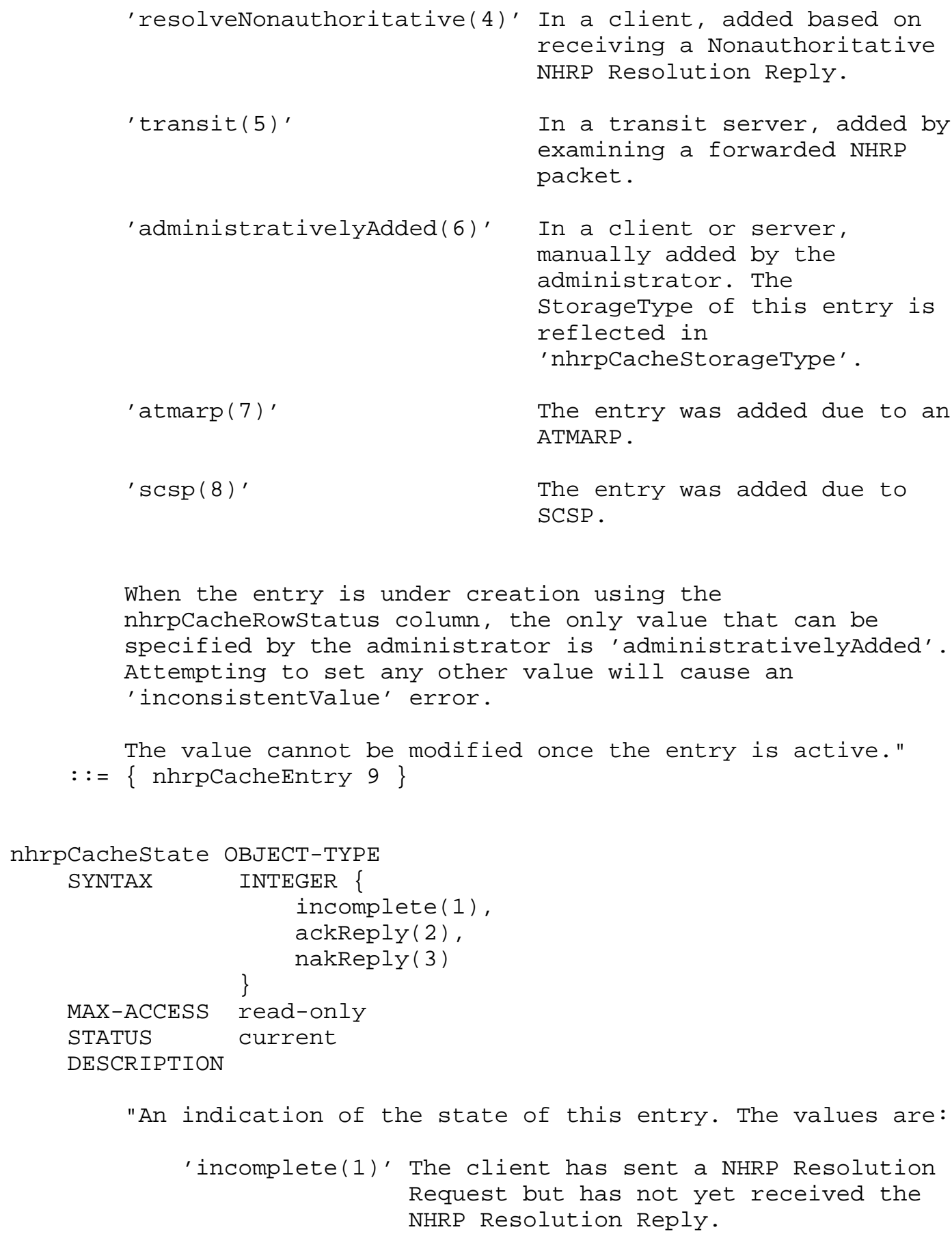




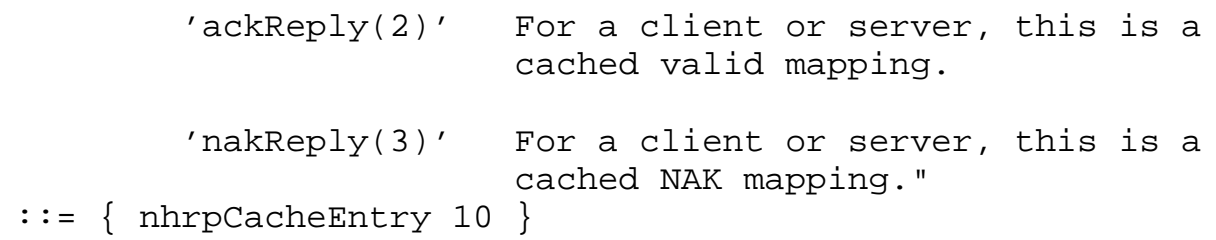




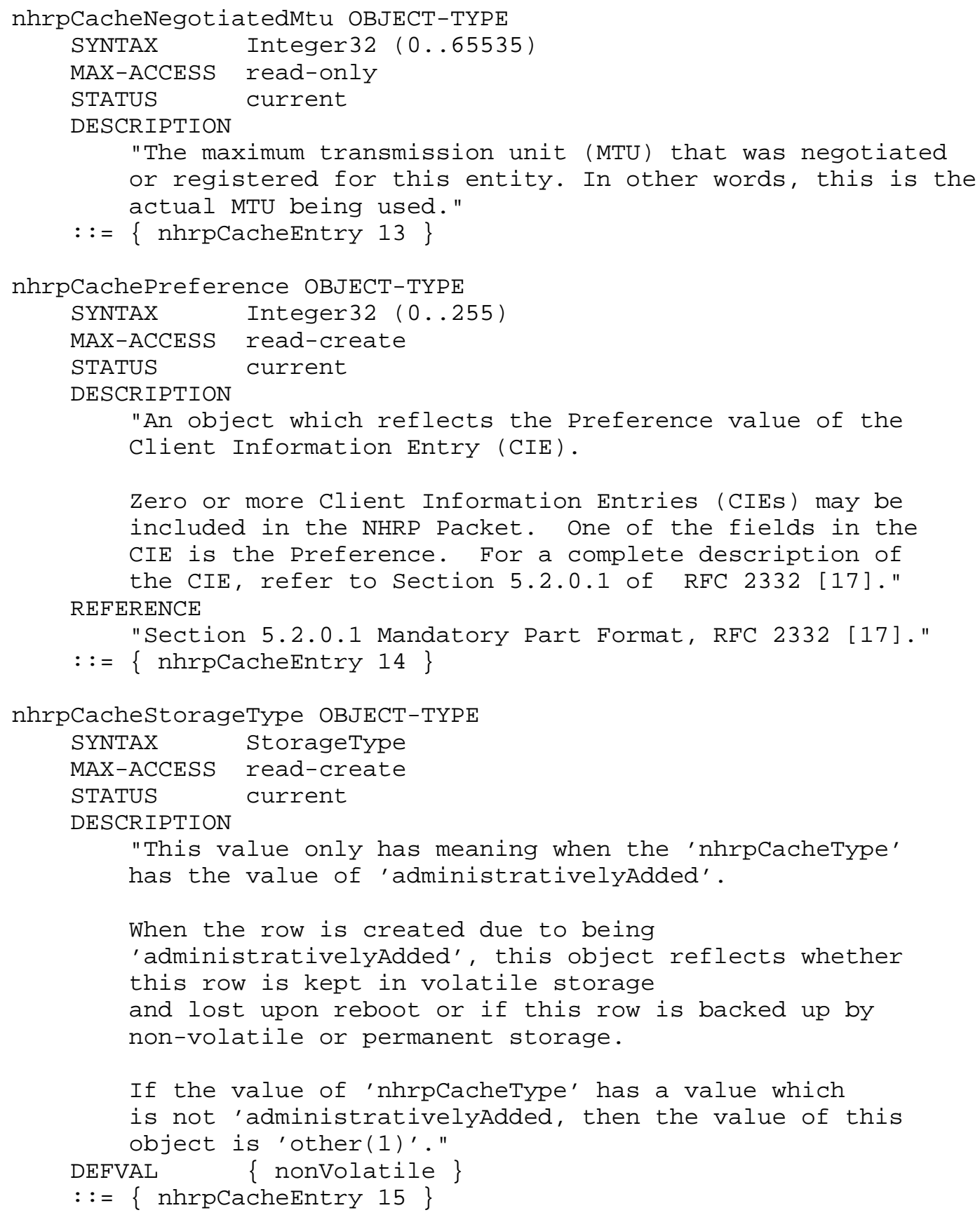




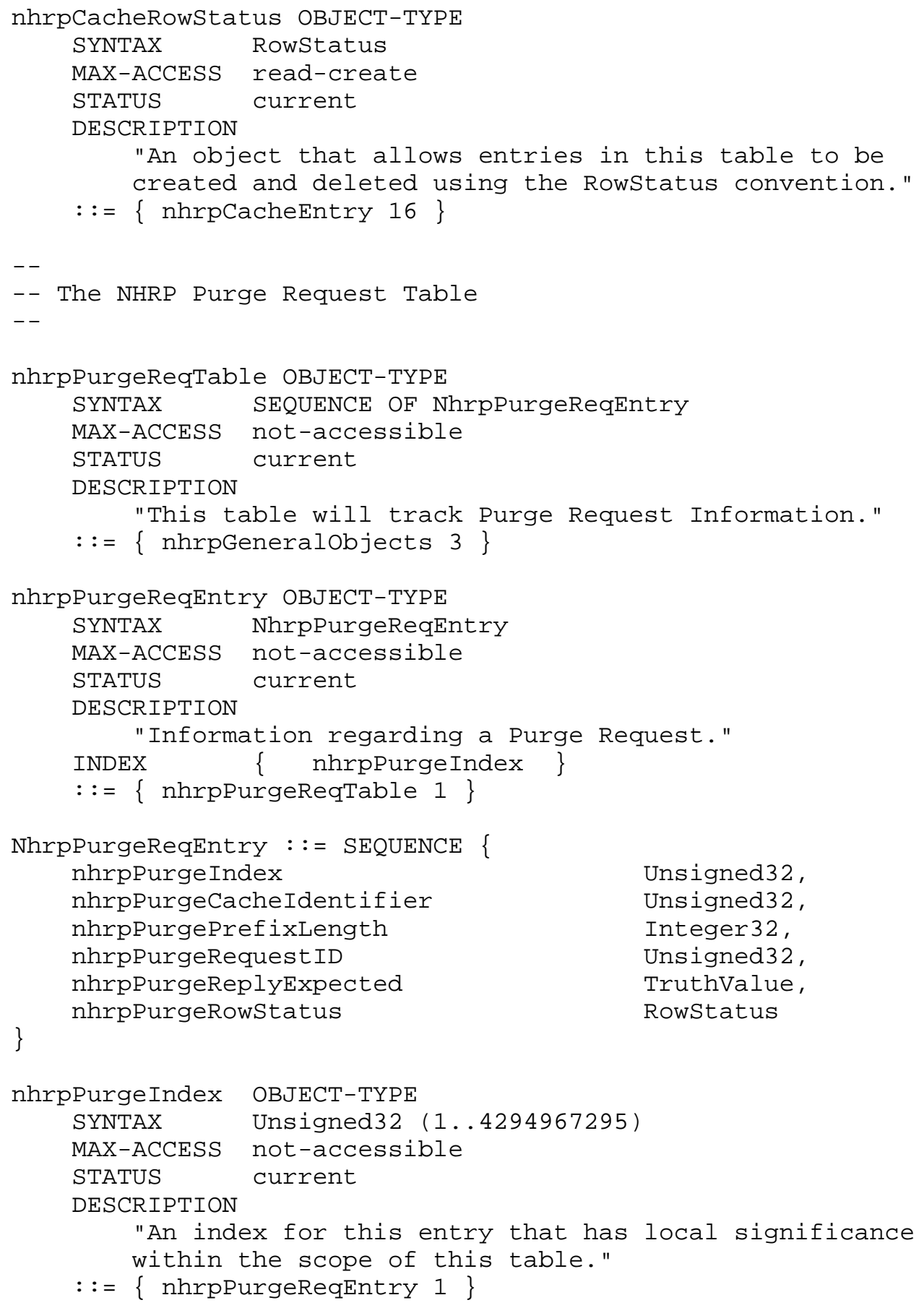




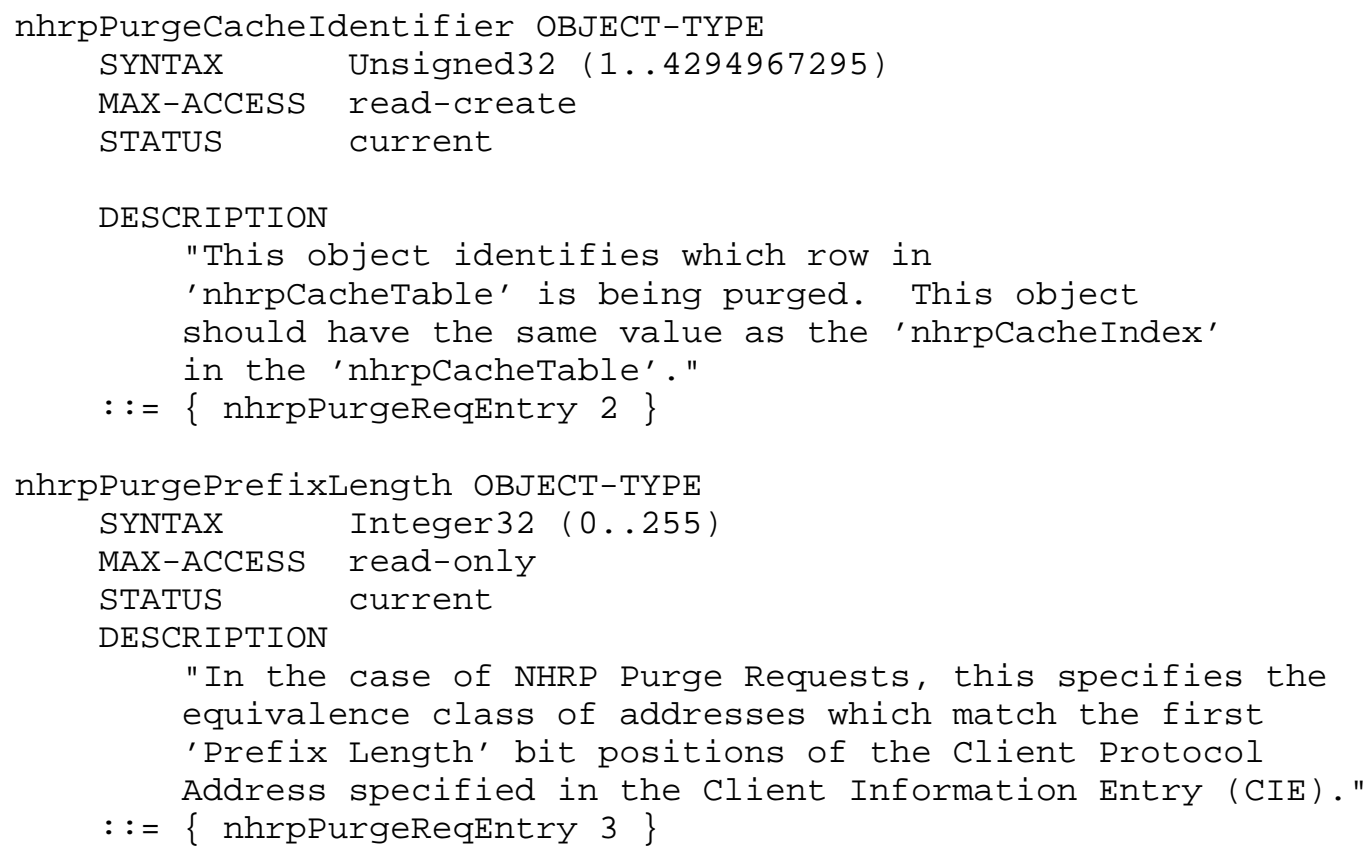




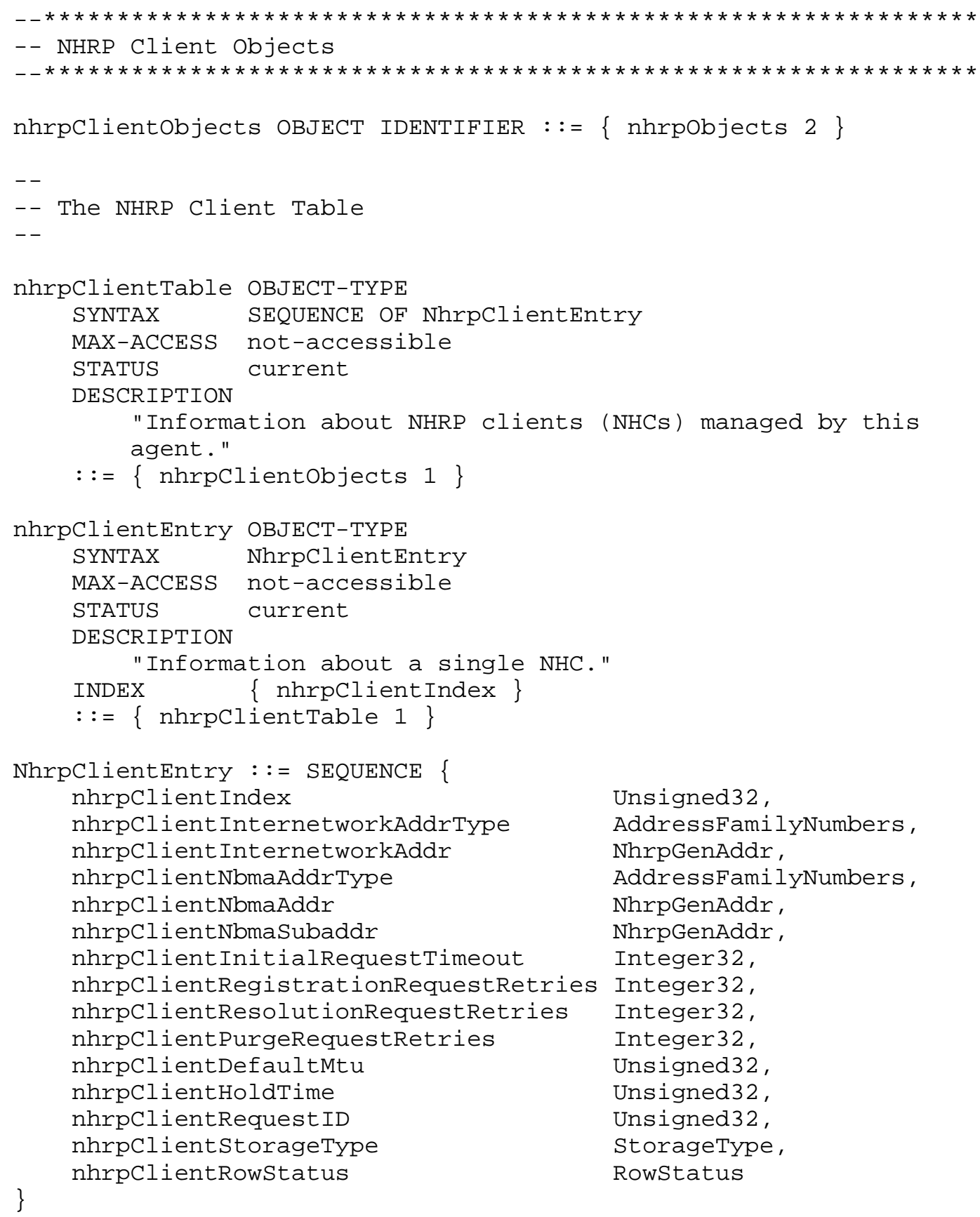




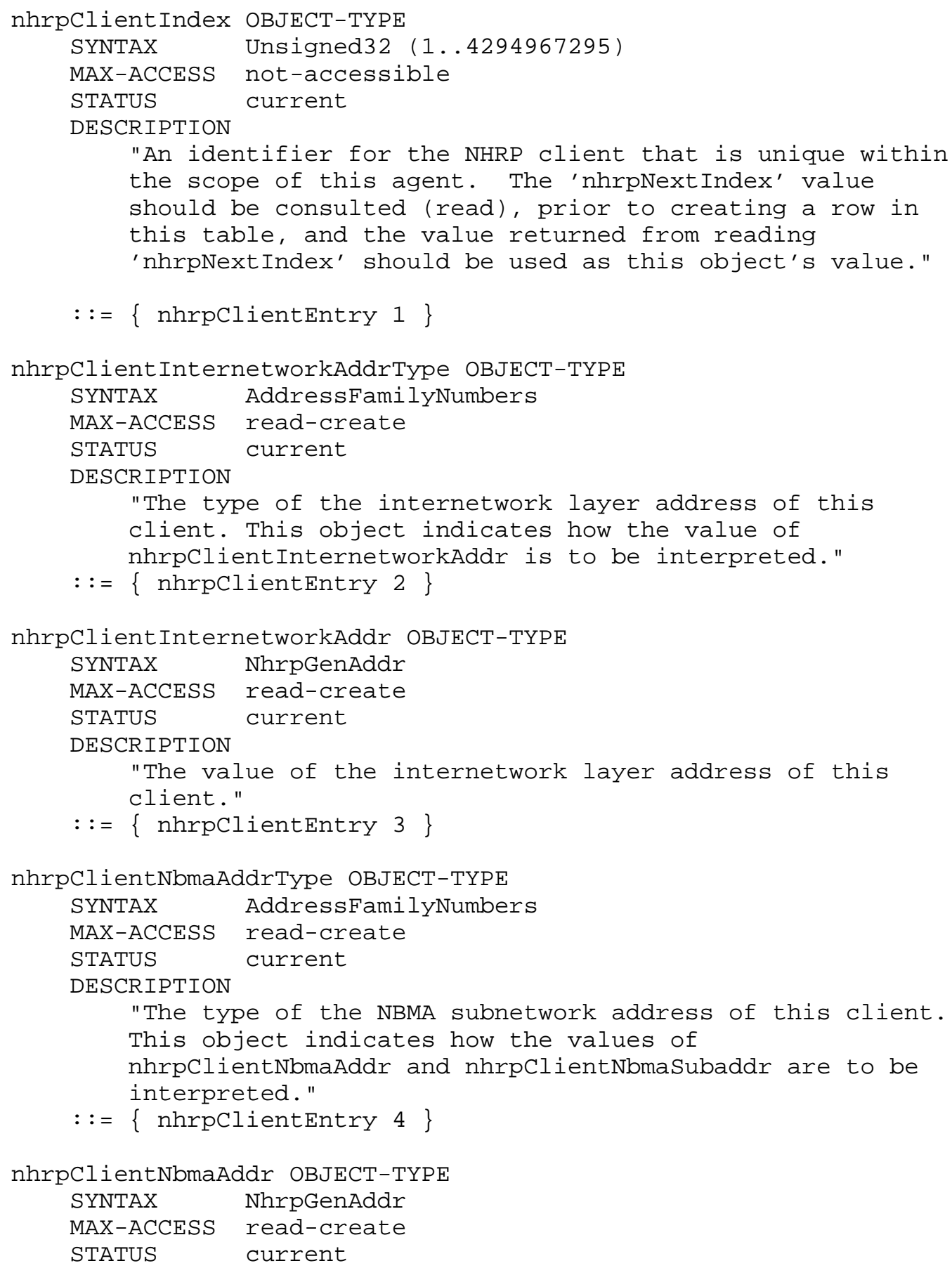




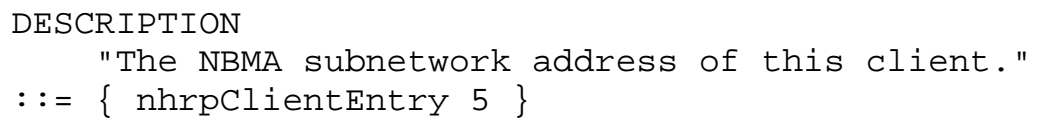




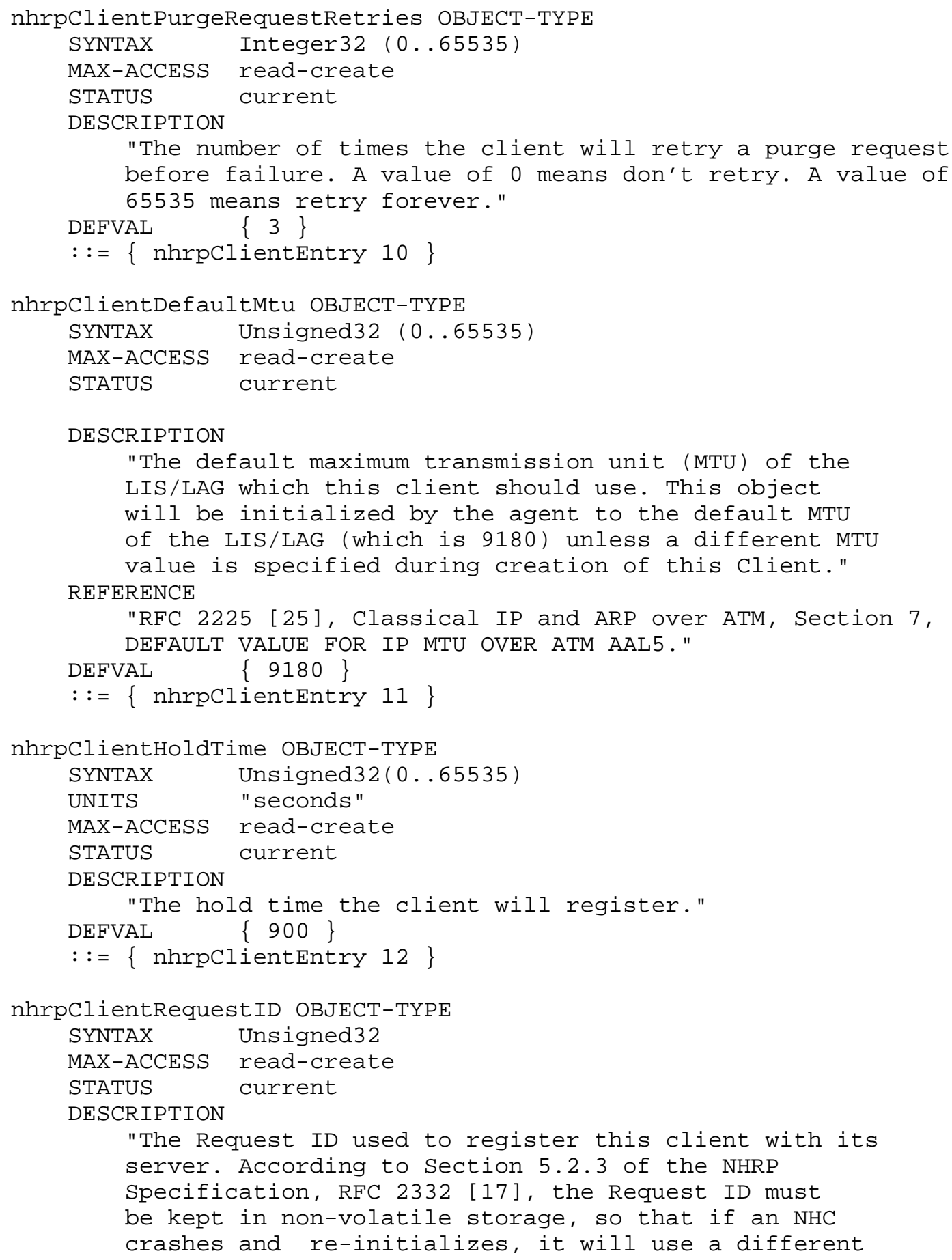




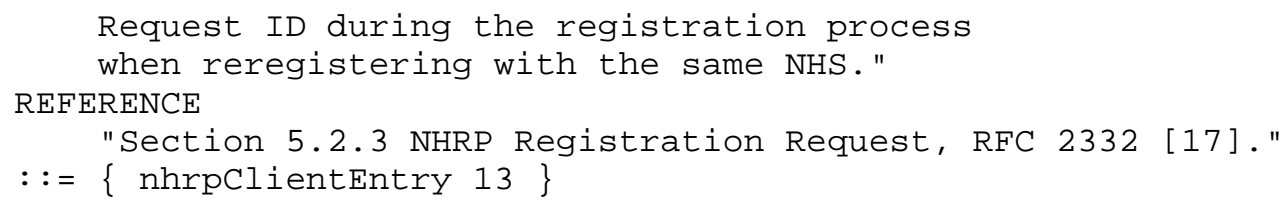


DESCRIPTION

"An NHC needs to maintain registration request information between the NHC and the NHS. An entry in this table represents information for a
INDEX $\{$ nhrpclientIndex, \} nhrpclientRegIndex

$::=\{$ nhrpclientRegistrationTable 1$\}$

NhrpClientRegistrationEntry : := SEQUENCE \{ nhrpclientRegIndex Unsigned32, nhrpClientRegUniqueness INTEGER, nhrpClientRegstate INTEGER, \} nhrpClientRegRowstatus Rowstatus

nhrpClientRegIndex OBJECT-TYPE

SYNTAX Unsigned32 (1..4294967295)

MAX-ACCESS not-accessible

STATUS current

DESCRIPTION

"An identifier for this entry such that it identifies a specific Registration Request from the NHC represented by the nhrpClientIndex."

$::=\{$ nhrpclientRegistrationEntry 1$\}$

nhrpClientRegUniqueness OBJECT-TYPE

SYNTAX INTEGER \{ requestunique (1), requestNotUnique (2)

\}

MAX-ACCESS read-create

STATUS current

DESCRIPTION

"The Uniqueness indicator for this Registration Request. If this object has the value of requestunique(1), then the Uniqueness bit is set in the the NHRP Registration Request represented by this row. The value cannot be changed once the row is created."

$::=\{$ nhrpclientRegistrationEntry 2$\}$

nhrpClientRegstate OBJECT-TYPE

SYNTAX INTEGER \{

other (1),

registering (2),

ackRegisterReply (3),

nakRegisterReply (4) 


$\begin{array}{ll} & \} \\ \text { MAX-ACCESS } & \text { read-only } \\ \text { STATUS } & \text { current } \\ \text { DESCRIPTION } & \end{array}$

"The registration state of this client. The values are:

'other(1)' The state of the registration request is not one of

'registering',

' ackRegisterReply' or

' nakRegisterReply'.

'registering(2)' $\begin{aligned} & \text { A registration request has } \\ & \text { been issued and a registration } \\ & \text { reply is expected. }\end{aligned}$
'ackRegisterReply (3)' $\quad \begin{aligned} & \text { A positive registration reply } \\ & \text { has been received. }\end{aligned}$
'nakRegisterReply (4)' $\quad \begin{aligned} & \text { The client has received a } \\ & \text { negative registration } \\ & \text { reply (NAK). " }\end{aligned}$

nhrpClientRegRowstatus OBJECT-TYPE

SYNTAX RowStatus

MAX-ACCESS read-create

STATUS current

DESCRIPTION

"An object that allows entries in this table to be created and deleted using the RowStatus convention." $::=\{$ nhrpclientRegistrationEntry 4$\}$

\section{$--$}

-- The NHRP Client->Server Table

$--$

nhrpClientNhsTable OBJECT-TYPE

SYNTAX SEQUENCE OF NhrpClientNhsEntry

MAX-ACCESS not-accessible

STATUS current

DESCRIPTION

"A table of NHSes that are available for use by this NHC (client). By default, the agent will add an entry to this table that corresponds to the client's default router." $::=\{$ nhrpclientobjects 3$\}$ 


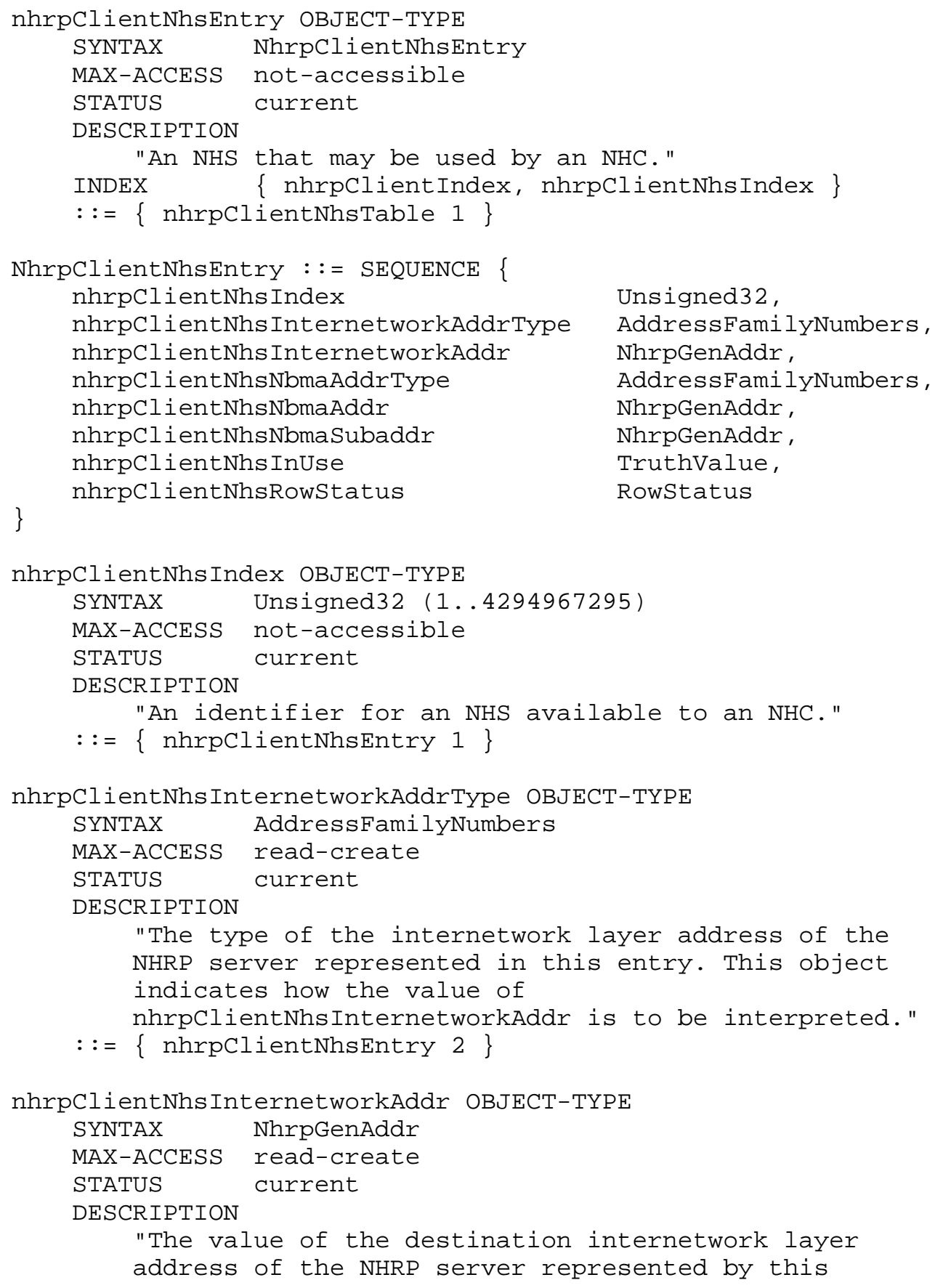




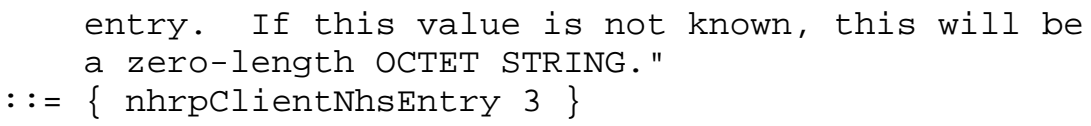




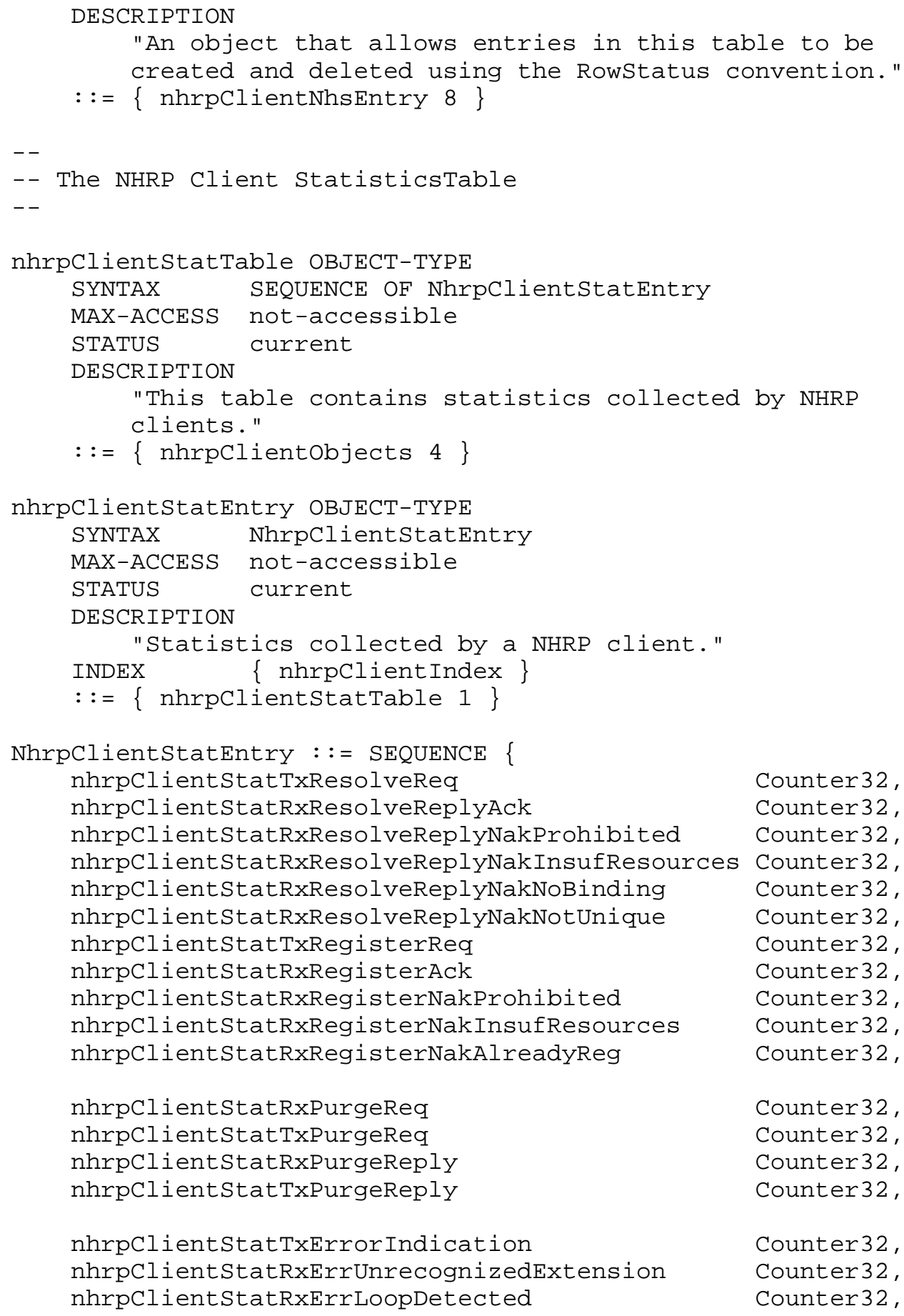




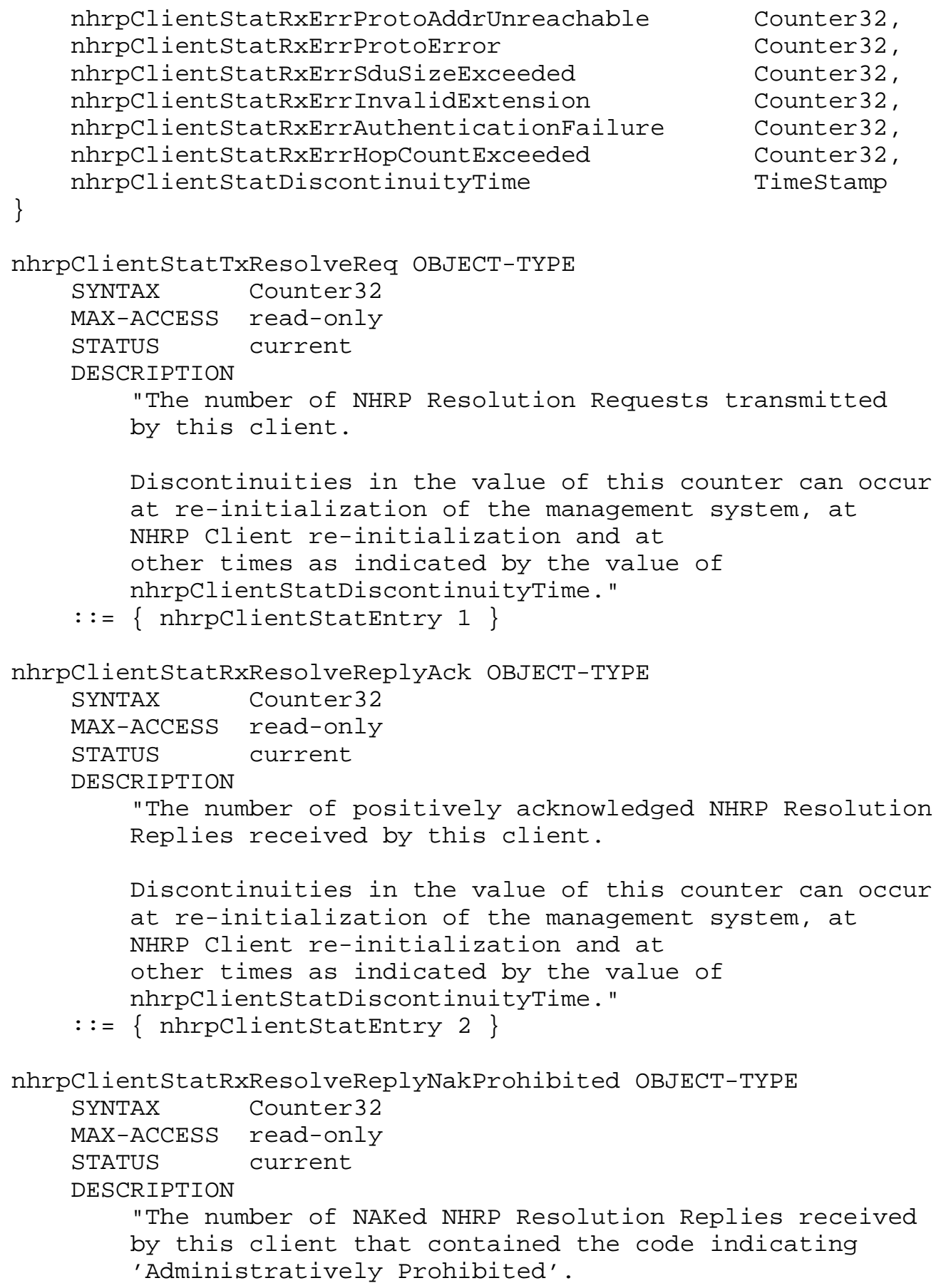




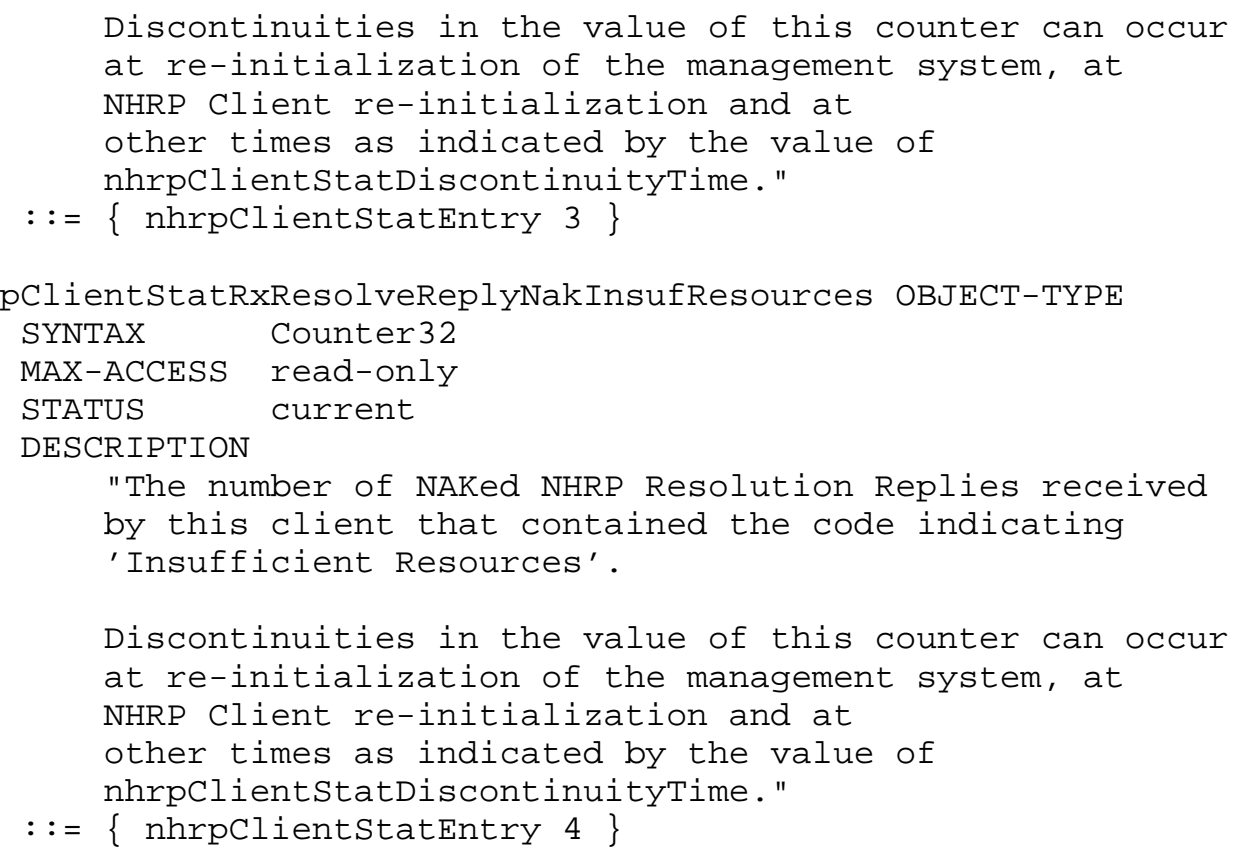

nhrpClientStatRxResolveReplyNakNoBinding OBJECT-TYPE

SYNTAX Counter32

MAX-ACCESS read-only

STATUS current

DESCRIPTION

"The number of NAKed NHRP Resolution Replies received by this client that contained the code indicating

'No Internetworking Layer Address to NBMA Address

Binding Exists'.

Discontinuities in the value of this counter can occur at re-initialization of the management system, at NHRP Client re-initialization and at other times as indicated by the value of nhrpClientStatDiscontinuityTime."

$::=\{$ nhrpclientStatEntry 5$\}$

nhrpClientStatRxResolveReplyNakNotUnique OBJECT-TYPE

SYNTAX Counter32

MAX-ACCESS read-only

STATUS current 
DESCRIPTION

"The number of NAKed NHRP Resolution Replies received

by this client that contained the code indicating

'Binding Exists But Is Not Unique'.

Discontinuities in the value of this counter can occur at re-initialization of the management system, at NHRP Client re-initialization and at other times as indicated by the value of nhrpclientstatDiscontinuityTime."

$::=\{$ nhrpclientstatEntry 6$\}$

nhrpClientStatTxRegisterReq OBJECT-TYPE

SYNTAX Counter32

MAX-ACCESS read-only

STATUS current

DESCRIPTION

"The number of NHRP Registration Requests transmitted

by this client.

Discontinuities in the value of this counter can occur at re-initialization of the management system, at NHRP Client re-initialization and at other times as indicated by the value of nhrpclientstatDiscontinuityTime."

$::=\{$ nhrpclientstatEntry 7$\}$

nhrpClientStatRxRegisterAck OBJECT-TYPE

SYNTAX Counter32

MAX-ACCESS read-only

STATUS current

DESCRIPTION

"The number of positively acknowledged NHRP Registration Replies received by this client.

Discontinuities in the value of this counter can occur at re-initialization of the management system, at NHRP Client re-initialization and at other times as indicated by the value of nhrpclientstatDiscontinuityTime."

$::=\{$ nhrpclientstatentry 8$\}$

nhrpClientStatRxRegisterNakProhibited OBJECT-TYPE

SYNTAX Counter32

MAX-ACCESS read-only

STATUS current 
DESCRIPTION

"The number of NAKed NHRP Registration Replies received

by this client that contained the code indicating

'Administratively Prohibited' .

Discontinuities in the value of this counter can occur at re-initialization of the management system, at NHRP Client re-initialization and at other times as indicated by the value of nhrpclientstatDiscontinuityTime."

$::=\{$ nhrpclientstatentry 9$\}$

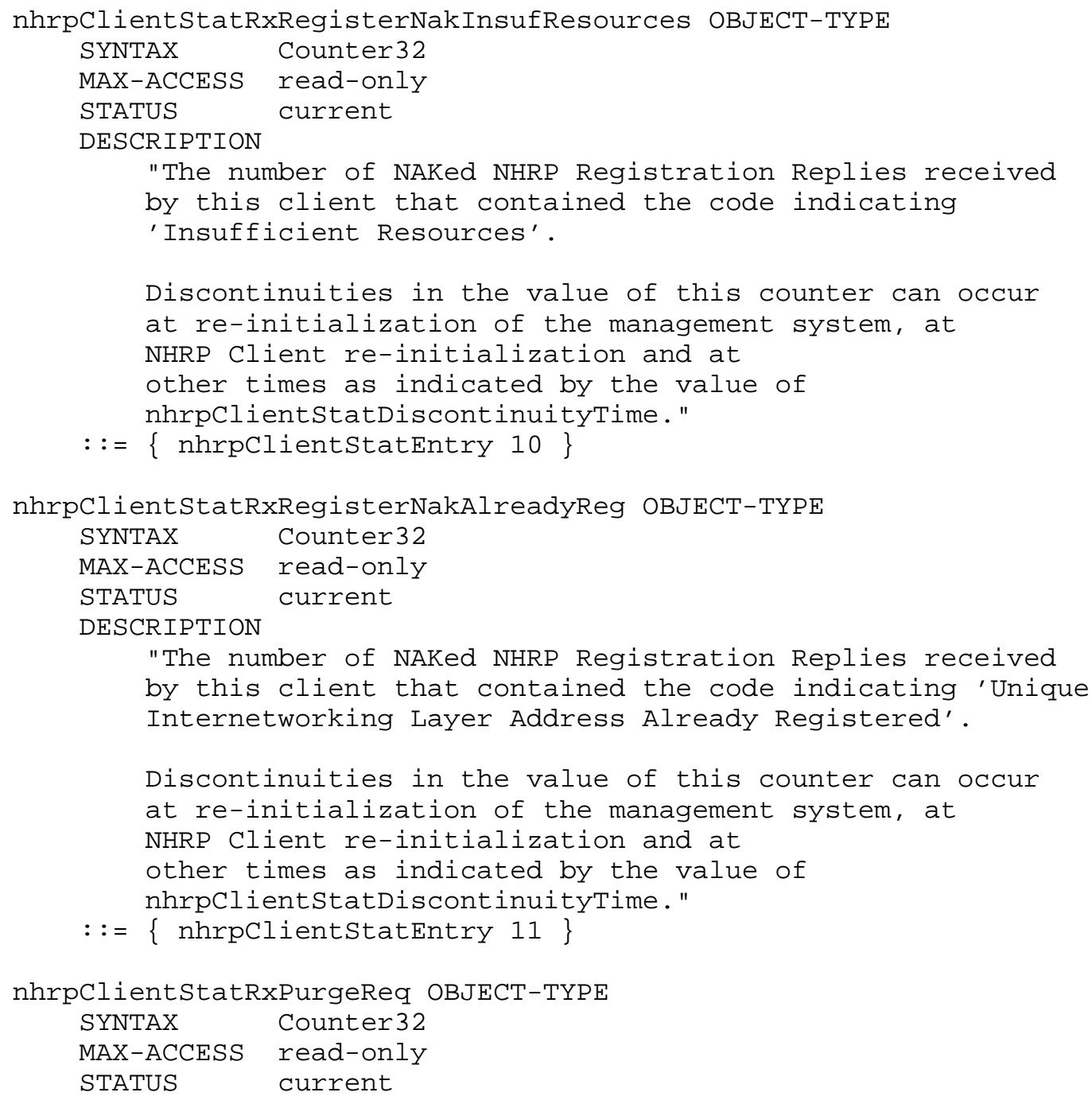


DESCRIPTION

"The number of NHRP Purge Requests received by this client.

Discontinuities in the value of this counter can occur at re-initialization of the management system, at NHRP Client re-initialization and at other times as indicated by the value of nhrpclientstatDiscontinuityTime."

$::=\{$ nhrpclientstatEntry 12$\}$

nhrpClientstatTxPurgeReq OBJECT-TYPE

SYNTAX Counter32

MAX-ACCESS read-only

STATUS current

DESCRIPTION

"The number of NHRP Purge Requests transmitted by this client.

Discontinuities in the value of this counter can occur at re-initialization of the management system, at NHRP Client re-initialization and at other times as indicated by the value of nhrpclientstatDiscontinuityTime."

$::=\{$ nhrpclientstatentry 13$\}$

nhrpClientStatRxPurgeReply OBJECT-TYPE

SYNTAX Counter32

MAX-ACCESS read-only

STATUS current

DESCRIPTION

"The number of NHRP Purge Replies received by this client.

Discontinuities in the value of this counter can occur at re-initialization of the management system, at NHRP Client re-initialization and at other times as indicated by the value of nhrpclientstatDiscontinuityTime."

$::=\{$ nhrpclientstatEntry 14$\}$

nhrpClientstatTxPurgeReply OBJECT-TYPE

SYNTAX Counter32

MAX-ACCESS read-only

STATUS current

DESCRIPTION

"The number of NHRP Purge Replies transmitted by this client. 


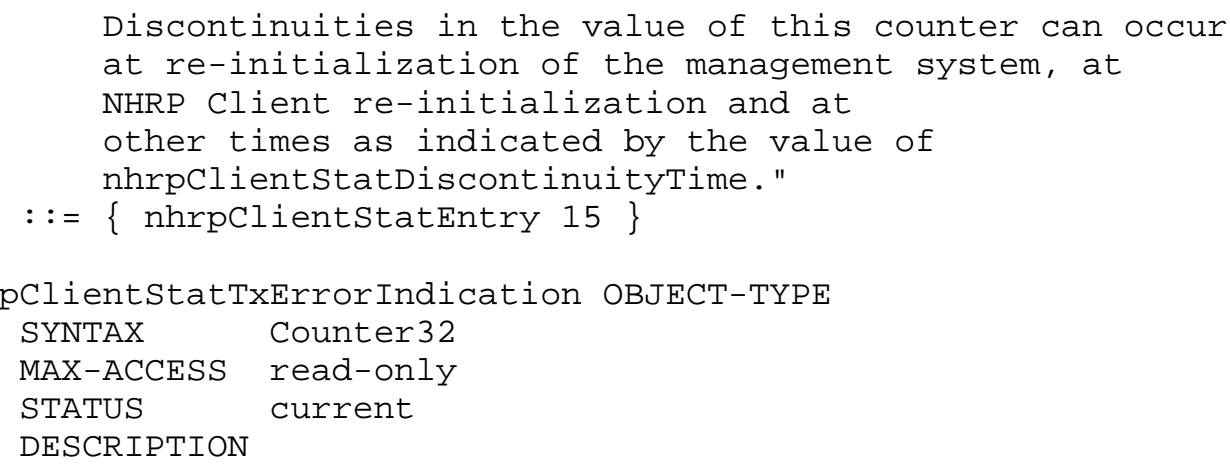

"The number of NHRP Error Indication packets transmitted by this client.

Discontinuities in the value of this counter can occur at re-initialization of the management system, at NHRP Client re-initialization and at other times as indicated by the value of nhrpclientstatDiscontinuityTime." REFERENCE

"Section 5.2.7 NHRP Error Indication, RFC 2332 [17]." $::=\{$ nhrpclientstatEntry 16$\}$

nhrpClientStatRxErrUnrecognizedExtension OBJECT-TYPE

SYNTAX Counter32

MAX-ACCESS read-only

STATUS current

DESCRIPTION

"The number of NHRP Error Indication packets received by this client with the error code

'Unrecognized Extension'.

Discontinuities in the value of this counter can occur at re-initialization of the management system, at NHRP Client re-initialization and at other times as indicated by the value of nhrpClientStatDiscontinuityTime." REFERENCE

"Section 5.2.7 NHRP Error Indication, RFC 2332 [17]." $::=\{$ nhrpclientstatEntry 17$\}$

nhrpClient StatRxErrLoopDetected OBJECT-TYPE

SYNTAX Counter32

MAX-ACCESS read-only

STATUS current 
DESCRIPTION

"The number of NHRP Error Indication packets received by this client with the error code 'NHRP Loop Detected'.

Discontinuities in the value of this counter can occur at re-initialization of the management system, at NHRP Client re-initialization and at other times as indicated by the value of nhrpClientstatDiscontinuityTime." REFERENCE

"Section 5.2.7 NHRP Error Indication, RFC 2332 [17]." $::=\{$ nhrpclientstatEntry 18$\}$

nhrpClientStatRxErrProtoAddrUnreachable OBJECT-TYPE SYNTAX Counter32

MAX-ACCESS read-only

STATUS current

DESCRIPTION

"The number of NHRP Error Indication packets received by this client with the error code 'Protocol Address Unreachable'.

Discontinuities in the value of this counter can occur at re-initialization of the management system, at NHRP Client re-initialization and at other times as indicated by the value of nhrpclientstatDiscontinuityTime." REFERENCE

"Section 5.2.7 NHRP Error Indication, RFC 2332 [17]." $::=\{$ nhrpclientstatEntry 19$\}$

nhrpClientStatRxErrProtoError OBJECT-TYPE SYNTAX Counter32

MAX-ACCESS read-only

STATUS current

DESCRIPTION

"The number of NHRP Error Indication packets received by this client with the error code 'Protocol Error'.

Discontinuities in the value of this counter can occur at re-initialization of the management system, at NHRP Client re-initialization and at other times as indicated by the value of nhrpClientstatDiscontinuityTime." REFERENCE

"Section 5.2.7 NHRP Error Indication, RFC 2332 [17]." $::=\{$ nhrpclientstatEntry 20$\}$ 


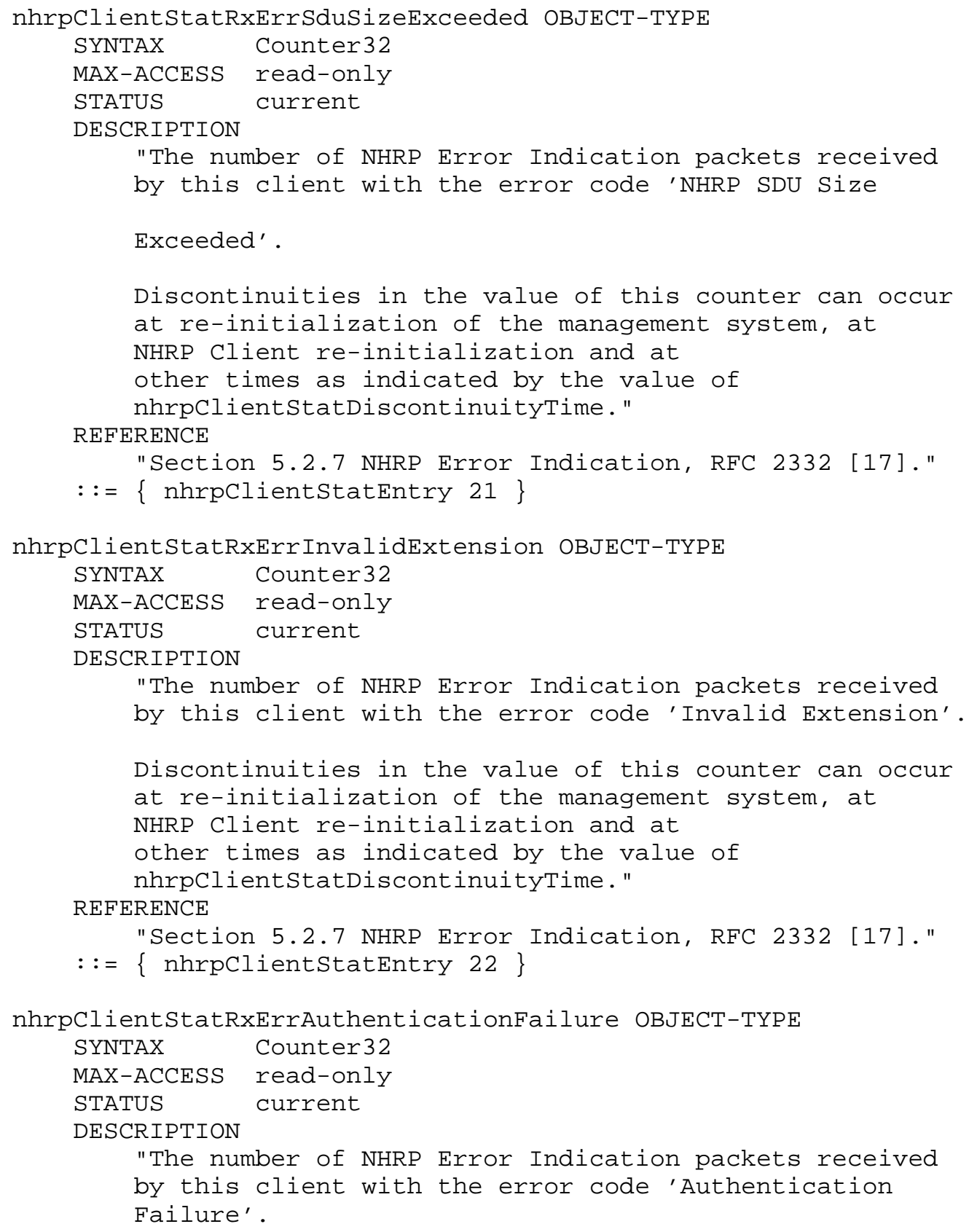




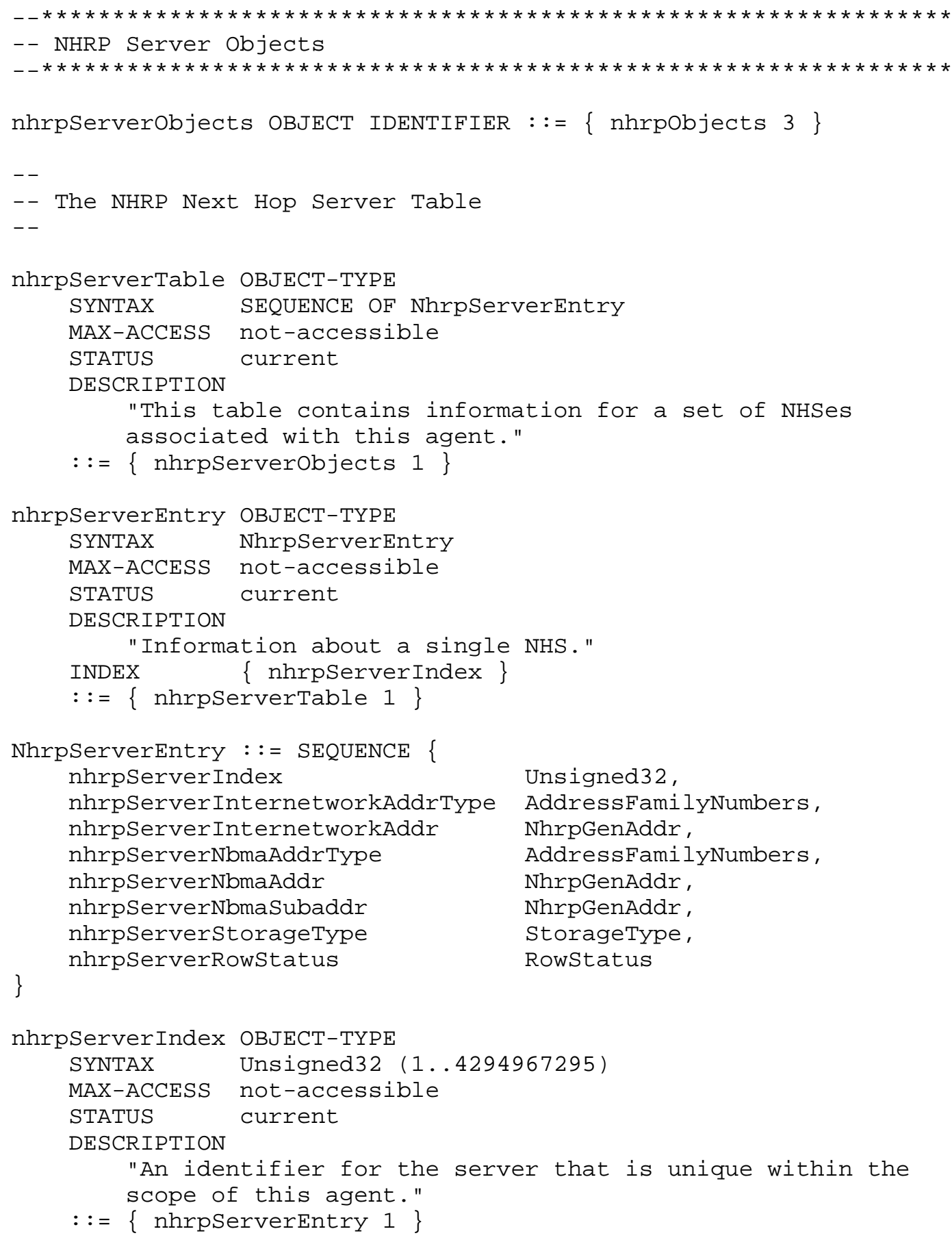




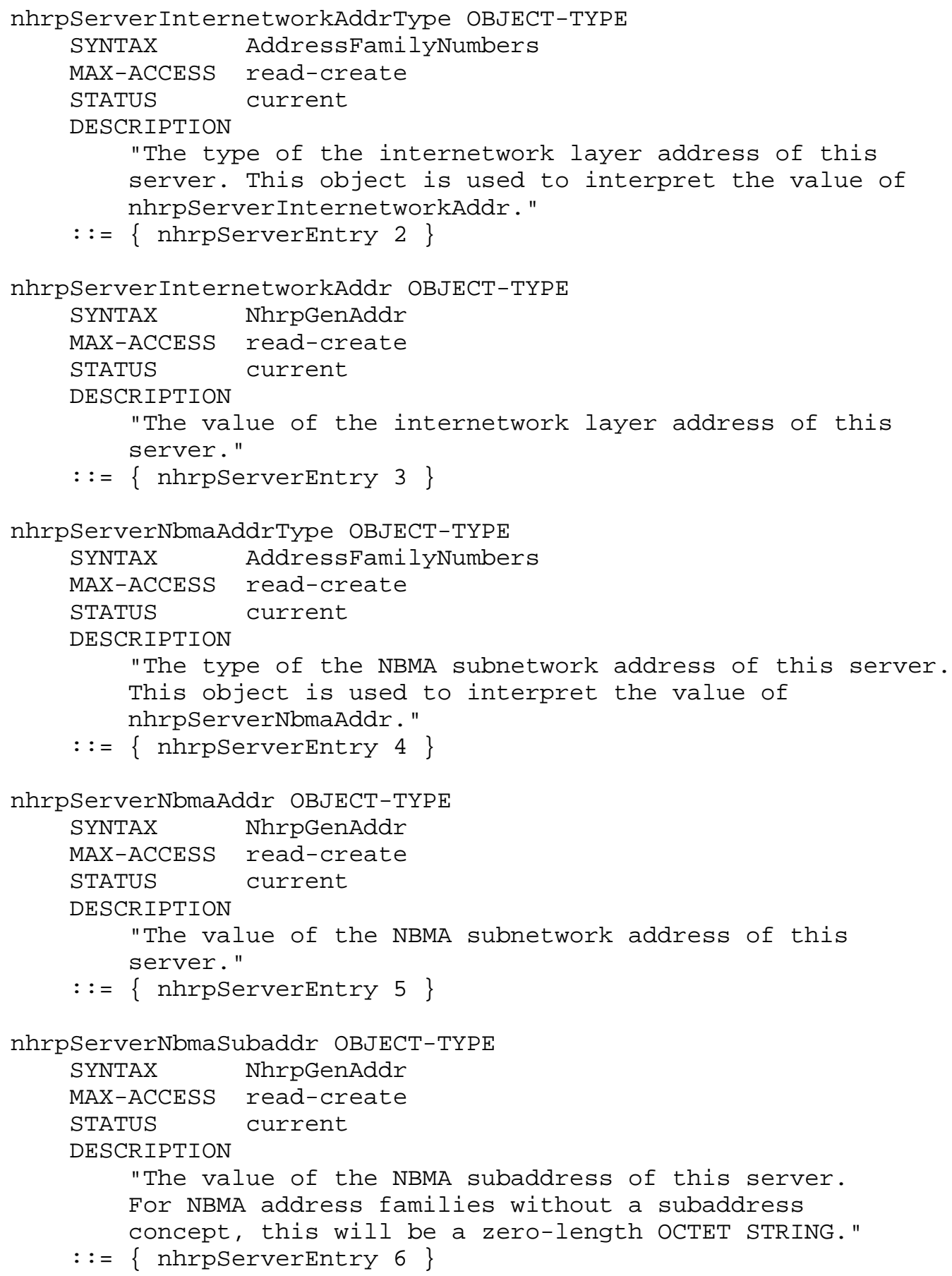




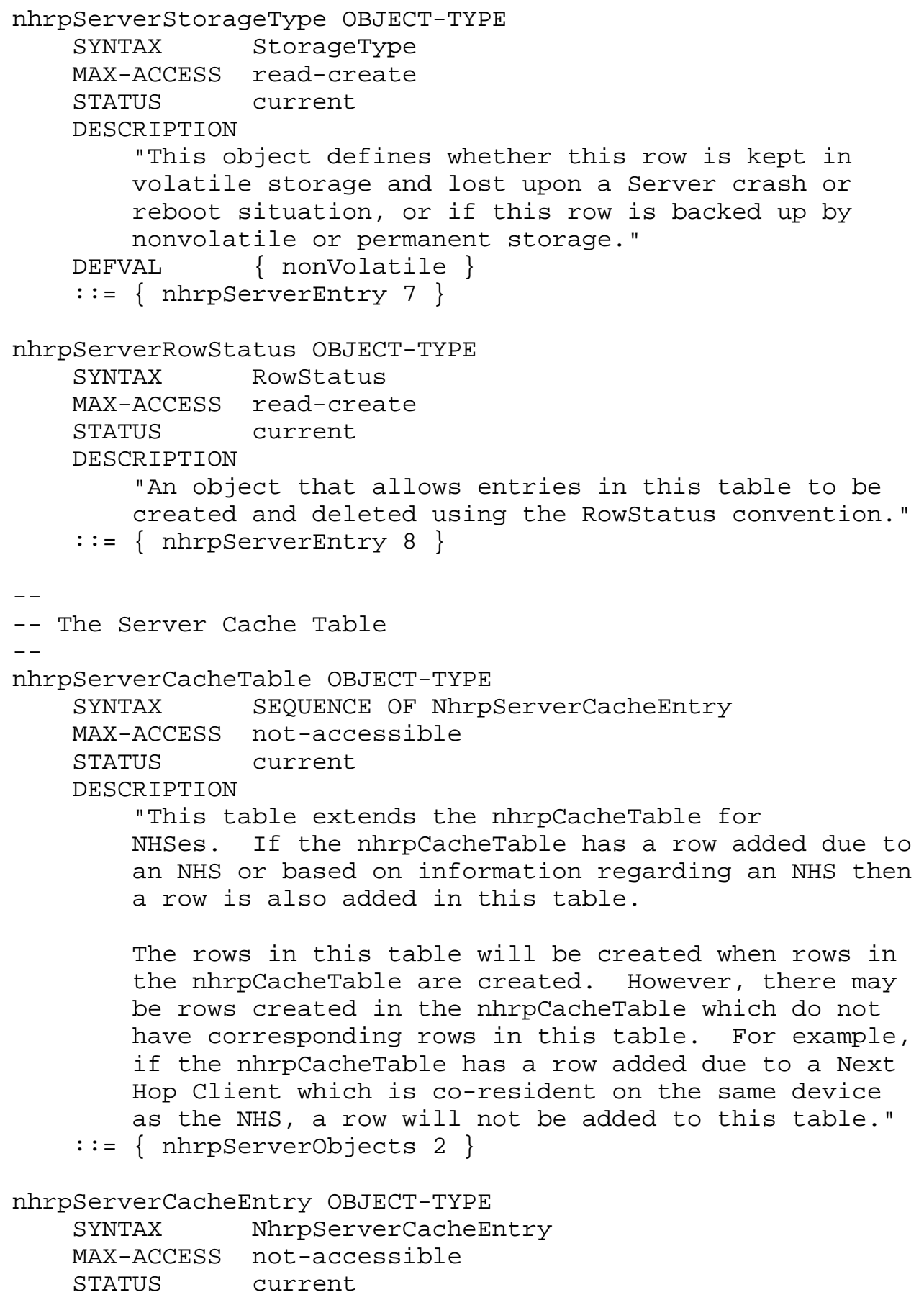




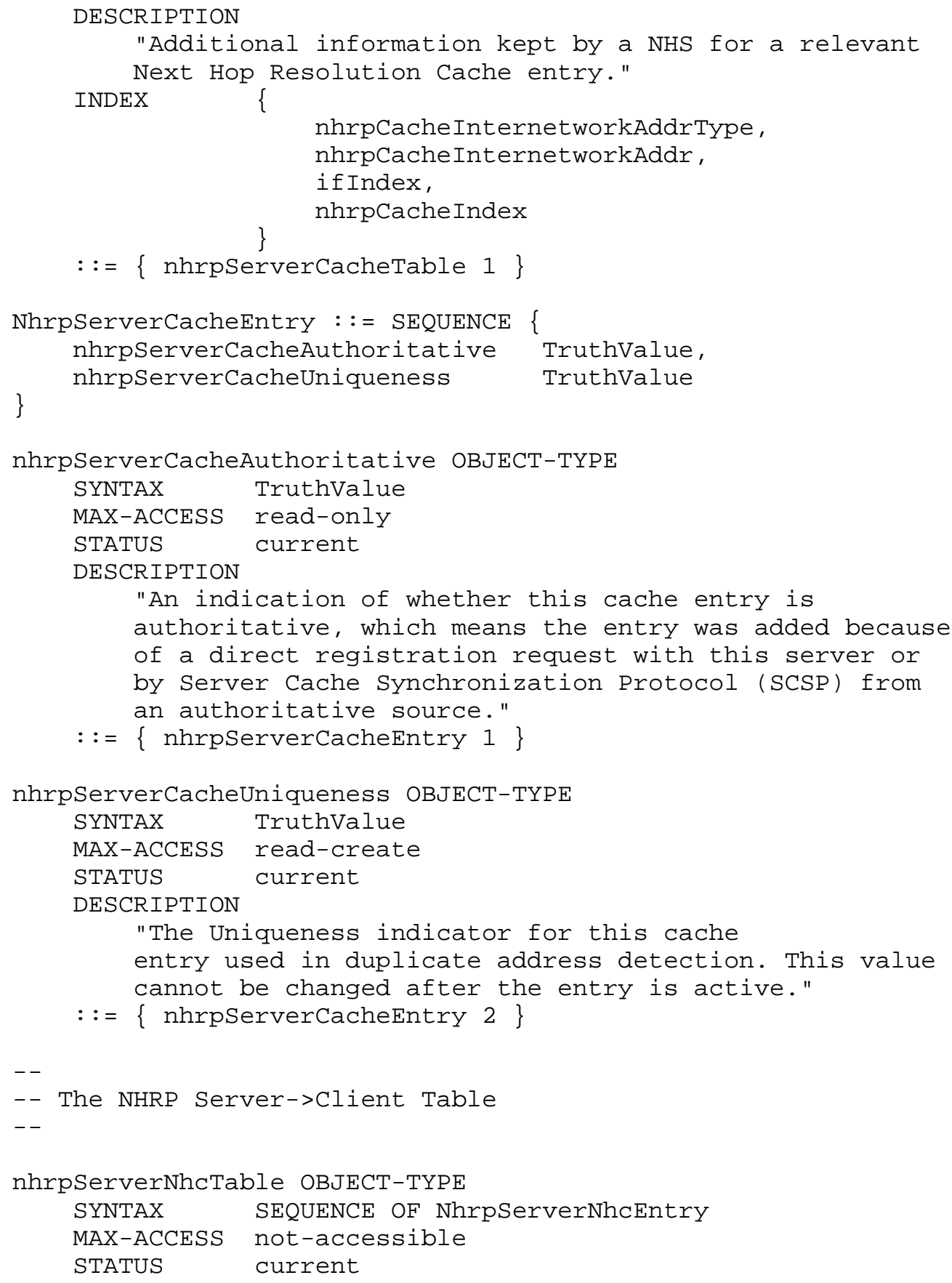


DESCRIPTION

"A table of NHCs that are available for use by this NHS (Server)."

REFERENCE

"Section 4 Configuration (Next Hop Servers), RFC 2332 [17]."

$::=\{$ nhrpserverObjects 3$\}$

nhrpserverNhcEntry OBJECT-TYPE

SYNTAX NhrpServerNhcEntry

MAX-ACCESS not-accessible

STATUS current

DESCRIPTION

"An NHC that may be used by an NHS."

INDEX \{ nhrpServerIndex, nhrpserverNhcIndex

$::=\{$ nhrpserverNhcTable 1$\}$

NhrpServerNhcEntry : := SEQUENCE \{

nhrpServerNhcIndex

Unsigned32,

nhrpServerNhcPrefixLength

Integer32,

nhrpServerNhcInternetworkAddrType

AddressFamilyNumbers,

nhrpServerNhcInternetworkAddr

NhrpGenAddr,

nhrpServerNhcNbmaAddrType

nhrpServerNhcNbmaAddr

AddressFamilyNumbers,

nhrpServerNhcNbmaSubaddr

NhrpGenAddr,

nhrpserverNhcInUse

NhrpGenAddr,

nhrpServerNhcRowstatus

Truthvalue.

\}

Rowstatus

nhrpServerNhcIndex OBJECT-TYPE

SYNTAX Unsigned32 (1..4294967295)

MAX-ACCESS not-accessible

STATUS current

DESCRIPTION

"An identifier for an NHC available to an NHS." $::=\{$ nhrpserverNhcEntry 1$\}$

nhrpServerNhcPrefixLength OBJECT-TYPE

SYNTAX Integer32 (0..255)

MAX-ACCESS read-create

STATUS current

DESCRIPTION

"The number of bits that define the internetwork

layer prefix associated with the nhrpServerNhcInternetworkAddr. "

$::=\{$ nhrpserverNhcEntry 2$\}$ 


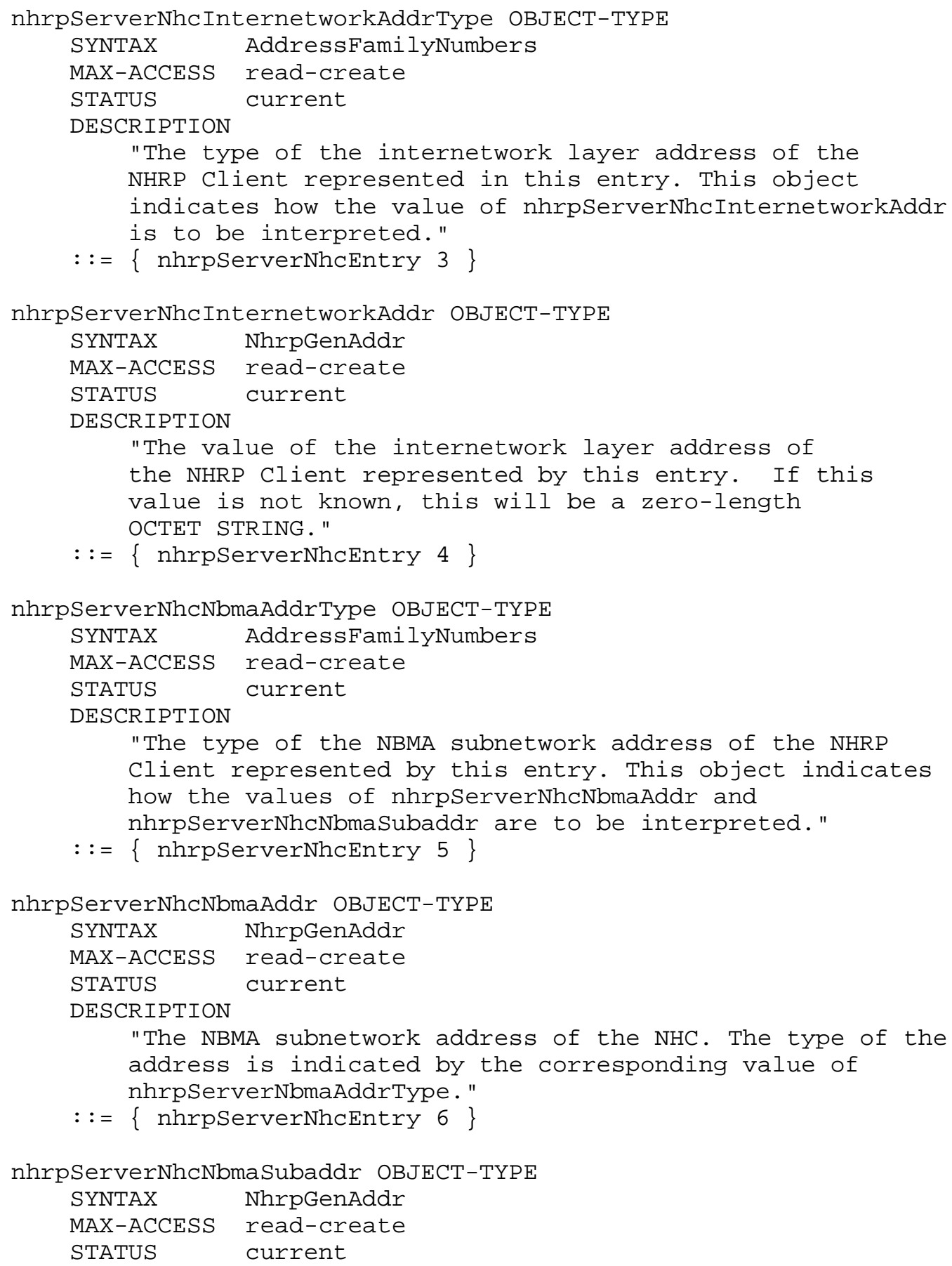


DESCRIPTION

"The NBMA subaddress of the NHC. For NMBA address familes that do not have the concept of subaddress, this will be a zero-length OCTET STRING."

$::=\{$ nhrpserverNhcEntry 7$\}$

nhrpServerNhcInUse OBJECT-TYPE

SYNTAX TruthValue

MAX-ACCESS read-only

STATUS current

DESCRIPTION

"An indication of whether this NHC is in use by the NHS." $::=\{$ nhrpserverNhcEntry 8$\}$

nhrpServerNhcRowStatus OBJECT-TYPE

SYNTAX Rowstatus

MAX-ACCESS read-create

STATUS current

DESCRIPTION

"An object that allows entries in this table to be created and deleted using the Rowstatus convention." $::=\{$ nhrpserverNhcEntry 9$\}$

$-$

-- The Next Hop Server Statistics Table

$--$

nhrpServerstatTable OBJECT-TYPE

SYNTAX SEQUENCE OF NhrpServerStatEntry

MAX-ACCESS not-accessible

STATUS current

DESCRIPTION

"Statistics collected by Next Hop Servers."

$::=\{$ nhrpserverobjects 4$\}$

nhrpServerstatEntry OBJECT-TYPE

SYNTAX NhrpserverstatEntry

MAX-ACCESS not-accessible

STATUS current

DESCRIPTION

"Statistics for a particular NHS. The statistics are

broken into received ( $\mathrm{Rx})$, transmitted ( $\mathrm{Tx}$ )

and forwarded $(\mathrm{FW})$. Forwarded (FW) would be done by a transit NHS."

INDEX \{ nhrpserverIndex \}

$::=\{$ nhrpserverStatTable 1$\}$ 
NhrpServerStatEntry : := SEQUENCE \{

nhrpServerStatRxResolveReq

Counter32, nhrpServerStat TxResolveReplyAck Counter32, nhrpServerStat TxResolveReplyNakProhibited counter32, nhrpServerStat TxResolveReplyNakInsufResources nhrpServerStat TxResolveReplyNakNoBinding nhrpServerStat TxResolveReplyNakNotUnique

counter 32 Counter32, Counter32,

nhrpServerStatRxRegisterReq nhrpServerStatTxRegisterAck nhrpServerStatTxRegisterNakProhibited nhrpServerStat TxRegisterNakInsufResources nhrpServerStat TxRegisterNakAlreadyReg

Counter32, Counter32, Counter32, Counter32, Counter32,

nhrpServerStatRxPurgeReq

Counter32, nhrpServerStat TxPurgeReq nhrpServerstatRxPurgeReply nhrpserverStatTxPurgeReply

Counter32,

Counter32,

Counter32,

-- Error Indications nhrpServerStatRxErrUnrecognizedExtension nhrpServerStatRxErrLoopDetected nhrpServerStatRxErrProtoAddrUnreachable nhrpserverStatRxErrProtoError nhrpServerStatRxErrSduSizeExceeded nhrpServerStatRxErrInvalidExtension nhrpServerStatRxErrInvalidResReplyReceived nhrpServerStatRxErrAuthenticationfailure nhrpServerStatRxErrHopCountExceeded

nhrpServerStat TxErrUnrecognizedExtension nhrpserverStat TxErrLoopDetected nhrpserverStat TxErrProtoAddrUnreachable nhrpserverStat TxErrProtoError nhrpServerStat TxErrSduSizeExceeded nhrpServerStat TxErrInvalidExtension nhrpserverStat TxErrAuthenticationfailure nhrpServerStat TxErrHopCountExceeded

Counter32, Counter32, Counter32, Counter32, Counter32, Counter32, Counter32, Counter32, Counter32,

Counter32, Counter32, Counter32, Counter32, Counter32, Counter32, Counter32, Counter32,

-- Transit NHS statistics nhrpServerStatFwResolveReq nhrpServerStatFwResolveReply nhrpServerStatFwRegisterReq nhrpServerStatFwRegisterReply nhrpserverStatFwPurgeReq nhrpServerstatFwPurgeReply nhrpserverStatFwErrorIndication nhrpserverstatDiscontinuityTime

Counter32, Counter32, Counter32, Counter32, Counter32, Counter32, Counter32, Timestamp 
\}

nhrpServerStatRxResolveReq OBJECT-TYPE

$\begin{array}{ll}\text { SYNTAX } & \text { Counter32 } \\ \text { MAX-ACCESS } & \text { read-only } \\ \text { STATUS } & \text { current }\end{array}$

DESCRIPTION

"The number of NHRP Resolution Requests received by this server.

Discontinuities in the value of this counter can occur at re-initialization of the management system, at NHRP Server re-initialization and at other times as indicated by the value of nhrpserverstatDiscontinuityTime."

$::=\{$ nhrpserverstatEntry 1$\}$

nhrpServerStatTxResolveReplyAck OBJECT-TYPE

SYNTAX Counter32

MAX-ACCESS read-only

STATUS current

DESCRIPTION

"The number of positively acknowledged NHRP

Resolution Replies transmitted by this server.

Discontinuities in the value of this counter can occur

at re-initialization of the management system, at

NHRP Server re-initialization and at

other times as indicated by the value of

nhrpserverstatDiscontinuityTime. "

$::=\{$ nhrpserverstatEntry 2$\}$

nhrpServerStatTxResolveReplyNakProhibited OBJECT-TYPE

SYNTAX Counter32

MAX-ACCESS read-only

STATUS current

DESCRIPTION

"The number of NAKed NHRP Resolution Replies

transmitted by this server with the code

'Administratively Prohibited'.

Discontinuities in the value of this counter can occur at re-initialization of the management system, at NHRP Server re-initialization and at

other times as indicated by the value of nhrpserverstatDiscontinuityTime."

$::=\{$ nhrpserverStatentry 3$\}$ 


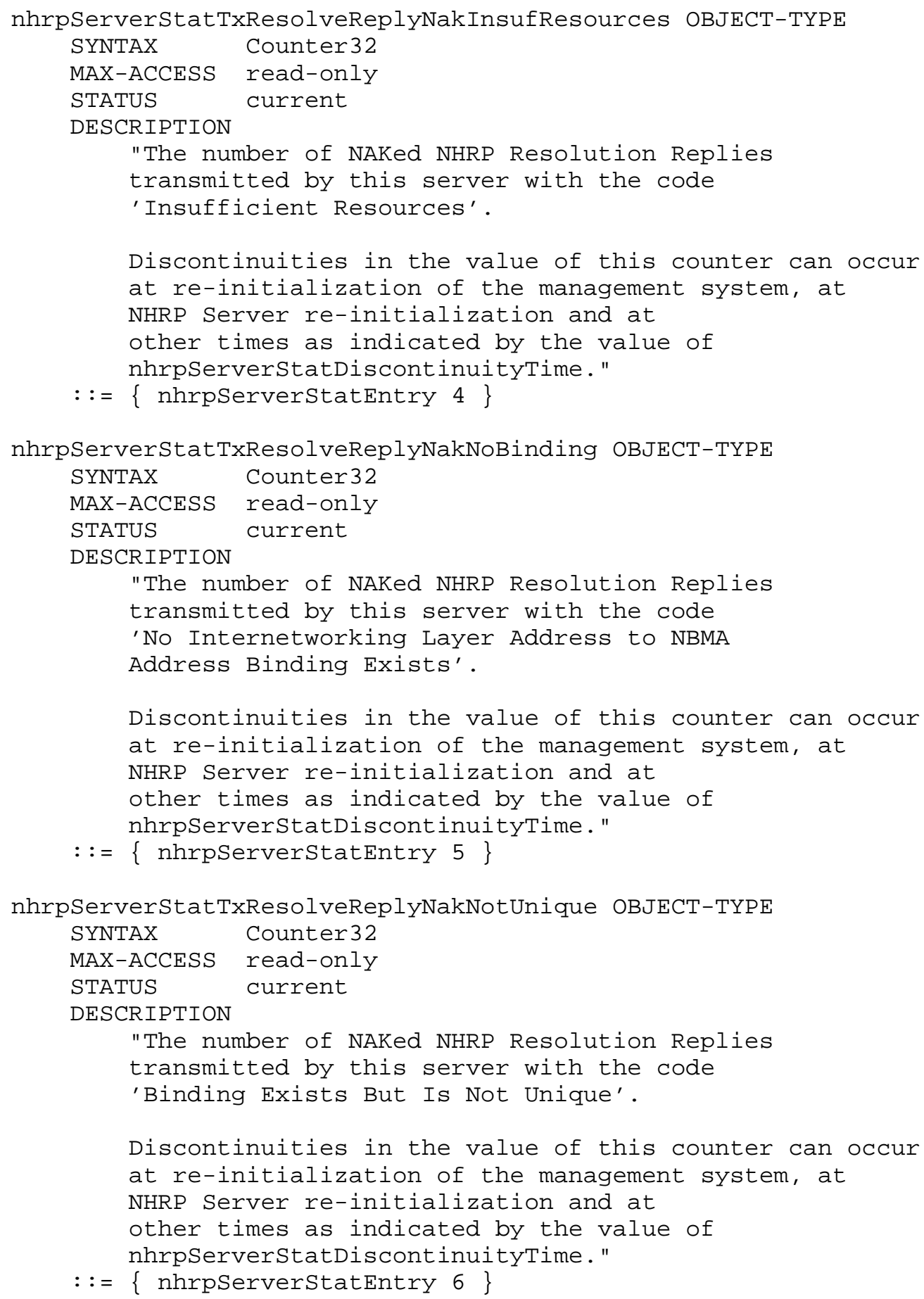




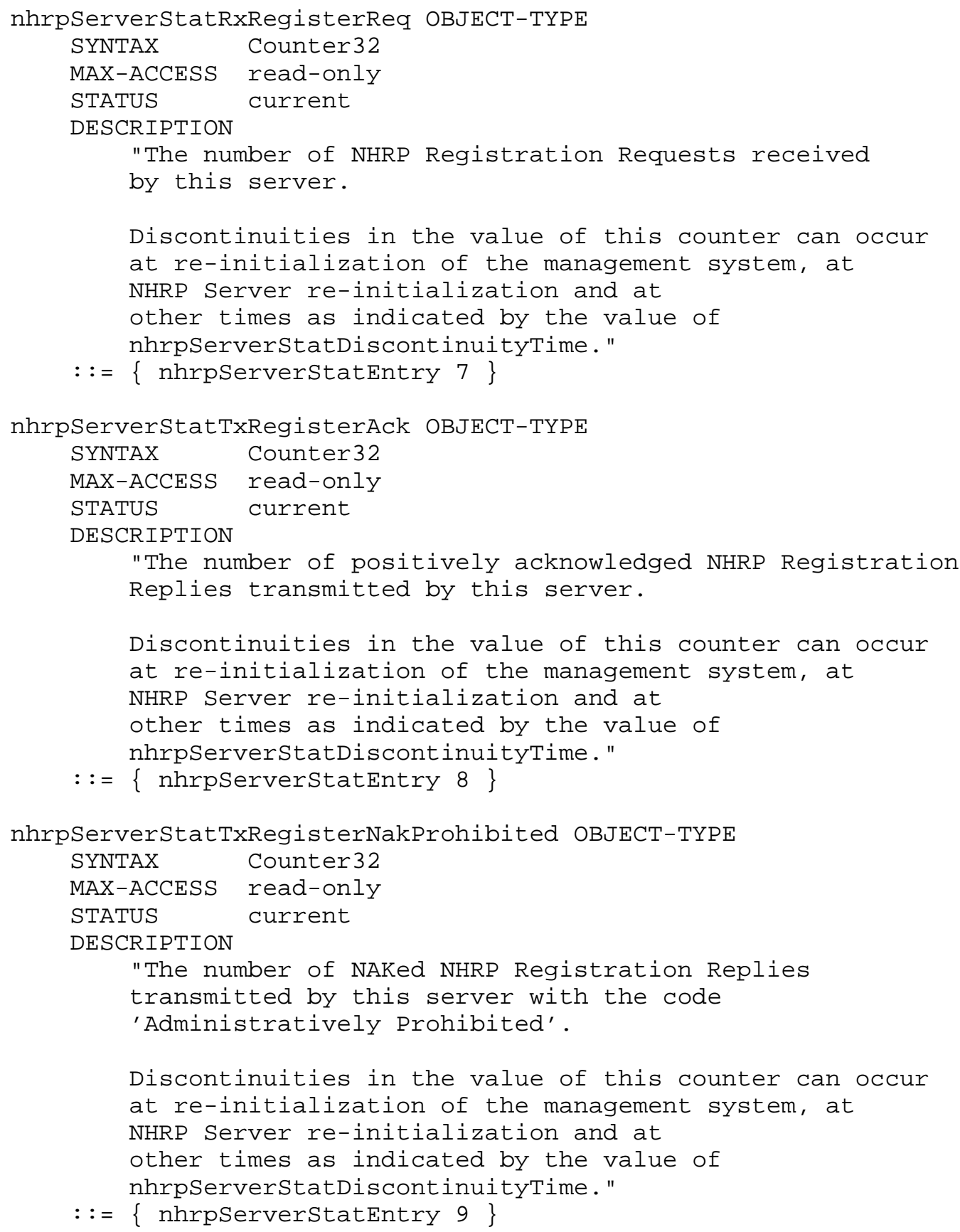




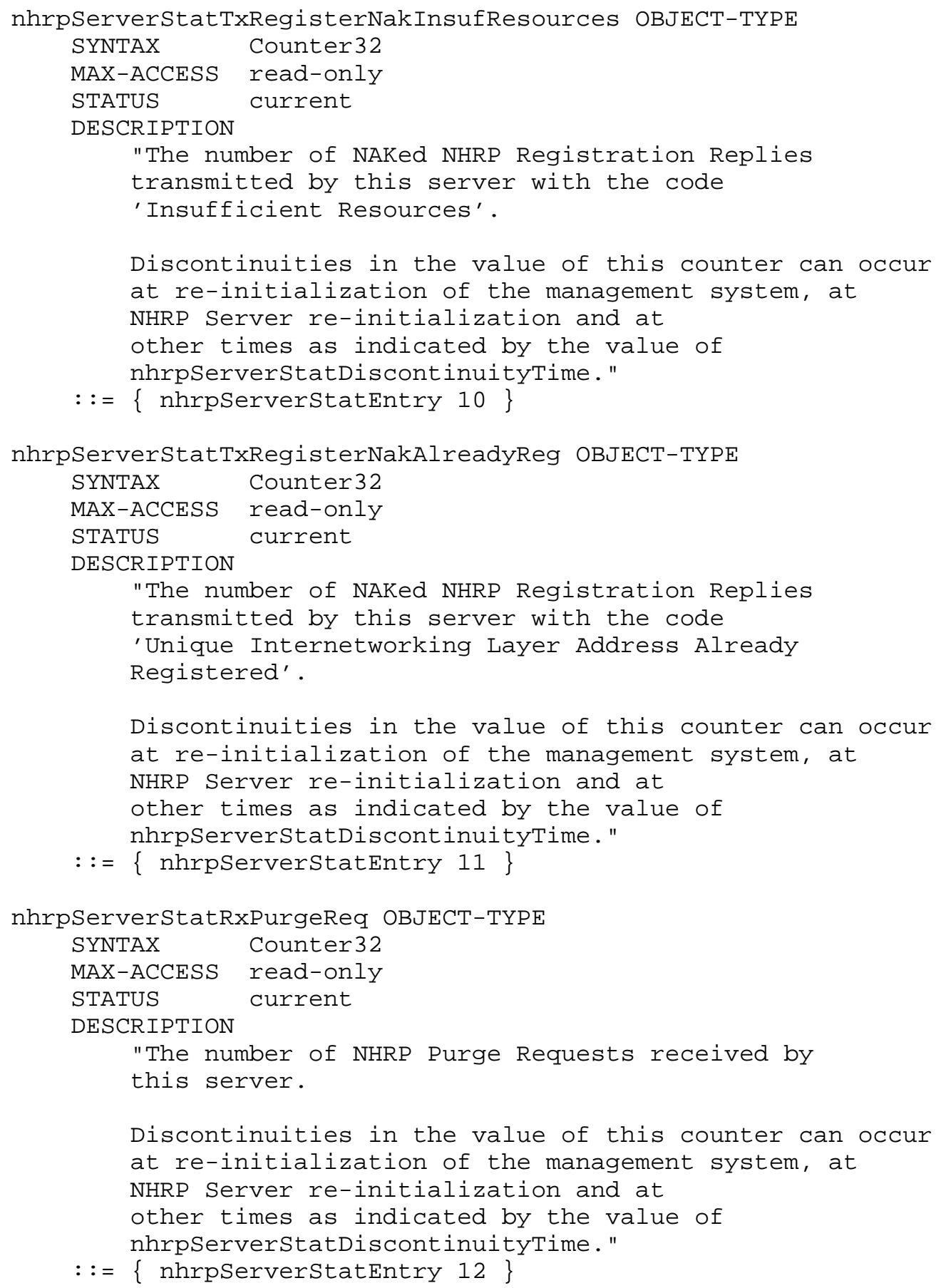




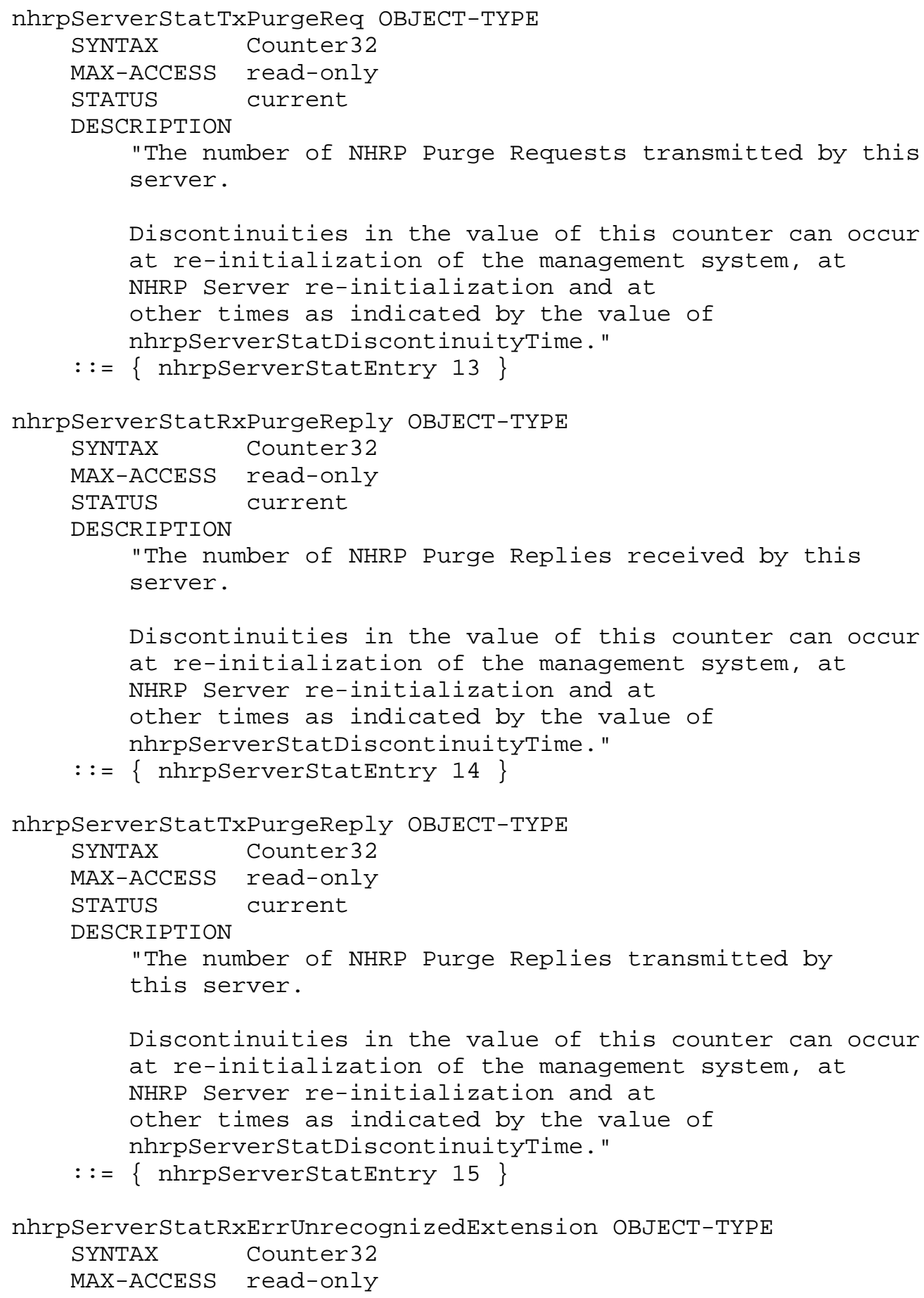




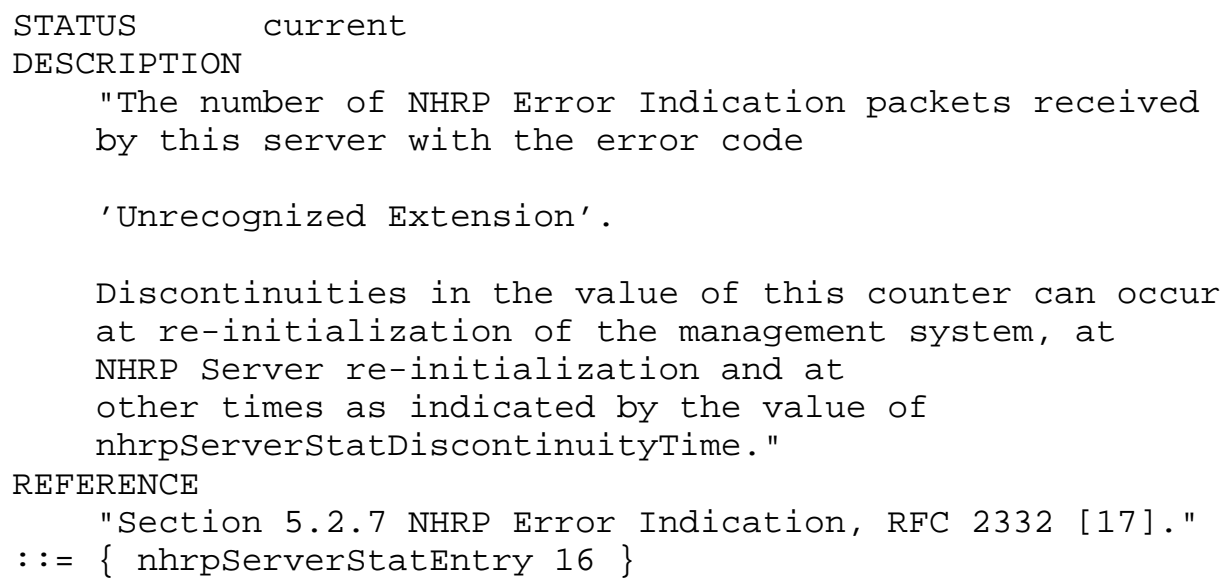


REFERENCE

"Section 5.2.7 NHRP Error Indication, RFC 2332 [17]." $::=\{$ nhrpserverstatEntry 18$\}$

nhrpServerStatRxErrProtoError OBJECT-TYPE

SYNTAX Counter32

MAX-ACCESS read-only

STATUS current

DESCRIPTION

"The number of NHRP Error Indication packets received by this server with the error code 'Protocol Error'.

Discontinuities in the value of this counter can occur at re-initialization of the management system, at NHRP Server re-initialization and at other times as indicated by the value of nhrpserverstatDiscontinuityTime." REFERENCE

"Section 5.2.7 NHRP Error Indication, RFC 2332 [17]." $::=\{$ nhrpserverstatentry 19$\}$

nhrpServerStatRxErrSduSizeExceeded OBJECT-TYPE

SYNTAX Counter32

MAX-ACCESS read-only

STATUS current

DESCRIPTION

"The number of NHRP Error Indication packets received by this server with the error code 'NHRP SDU Size Exceeded'.

Discontinuities in the value of this counter can occur at re-initialization of the management system, at NHRP Server re-initialization and at other times as indicated by the value of nhrpserverstatDiscontinuityTime." REFERENCE

"Section 5.2.7 NHRP Error Indication, RFC 2332 [17]." $::=\{$ nhrpserverStatEntry 20$\}$

nhrpServerStatRxErrInvalidExtension OBJECT-TYPE

SYNTAX Counter32

MAX-ACCESS read-only

STATUS current 
DESCRIPTION

"The number of NHRP Error Indication packets received

by this server with the error code 'Invalid Extension'.

Discontinuities in the value of this counter can occur at re-initialization of the management system, at NHRP Server re-initialization and at other times as indicated by the value of nhrpserverstatDiscontinuityTime." REFERENCE

"Section 5.2.7 NHRP Error Indication, RFC 2332 [17]." $::=\{$ nhrpserverstatEntry 21$\}$

nhrpServerStatRxErrInvalidResReplyReceived OBJECT-TYPE

SYNTAX Counter32

MAX-ACCESS read-only

STATUS current

DESCRIPTION

"The number of NHRP Error Indication packets received by this server with the error code 'Invalid Resolution Reply Received'.

Discontinuities in the value of this counter can occur at re-initialization of the management system, at NHRP Server re-initialization and at other times as indicated by the value of nhrpserverstatDiscontinuityTime." REFERENCE

"Section 5.2.7 NHRP Error Indication, RFC 2332 [17]." $::=\{$ nhrpserverstatEntry 22$\}$

nhrpServerStatRxErrAuthenticationFailure OBJECT-TYPE SYNTAX Counter32

MAX-ACCESS read-only

STATUS current

DESCRIPTION

"The number of NHRP Error Indication packets received by this server with the error code

'Authentication Failure'.

Discontinuities in the value of this counter can occur at re-initialization of the management system, at NHRP Server re-initialization and at other times as indicated by the value of nhrpserverstatDiscontinuityTime."

REFERENCE

"Section 5.2.7 NHRP Error Indication, RFC 2332 [17]." $::=\{$ nhrpserverstatEntry 23$\}$ 


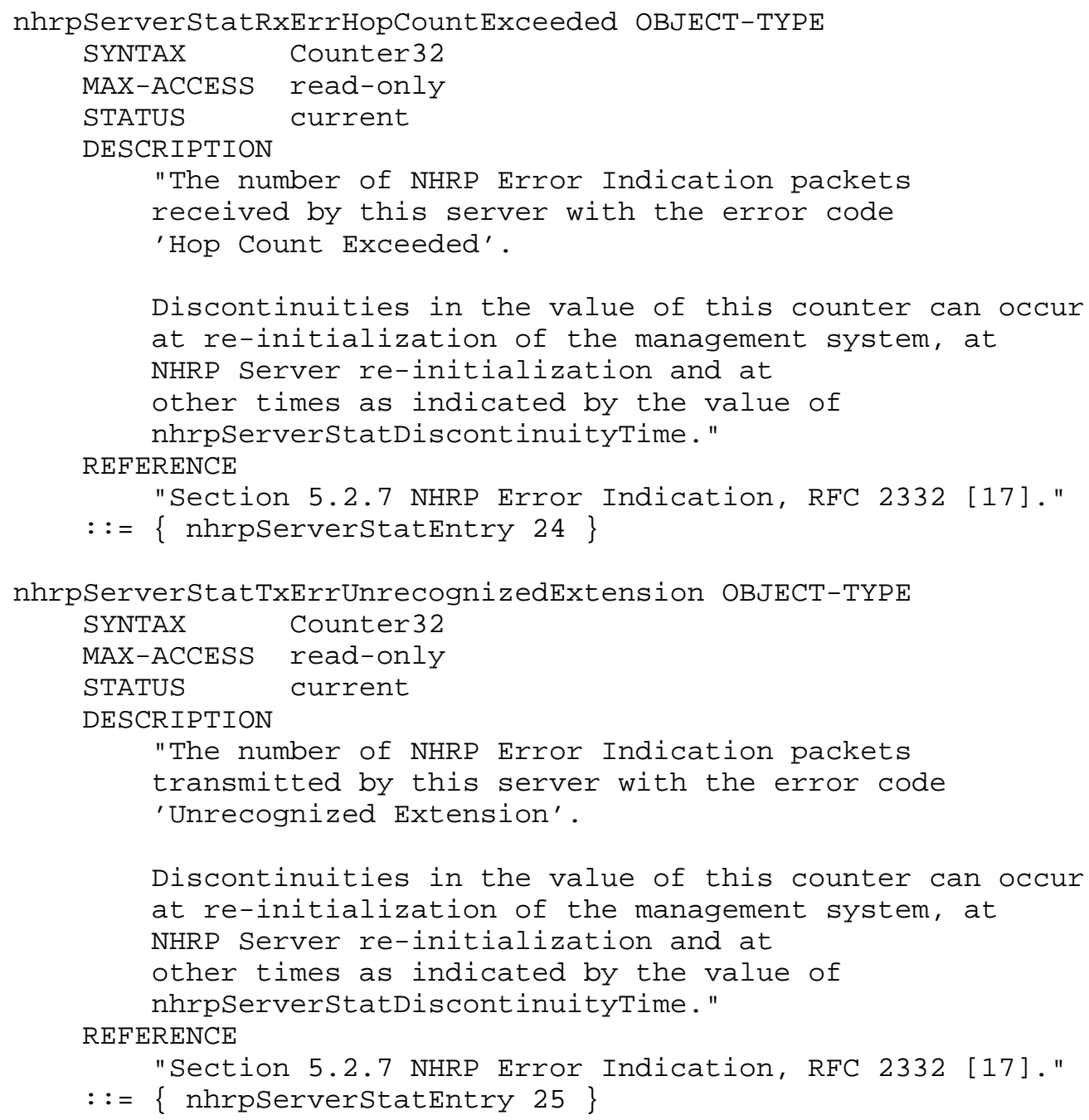




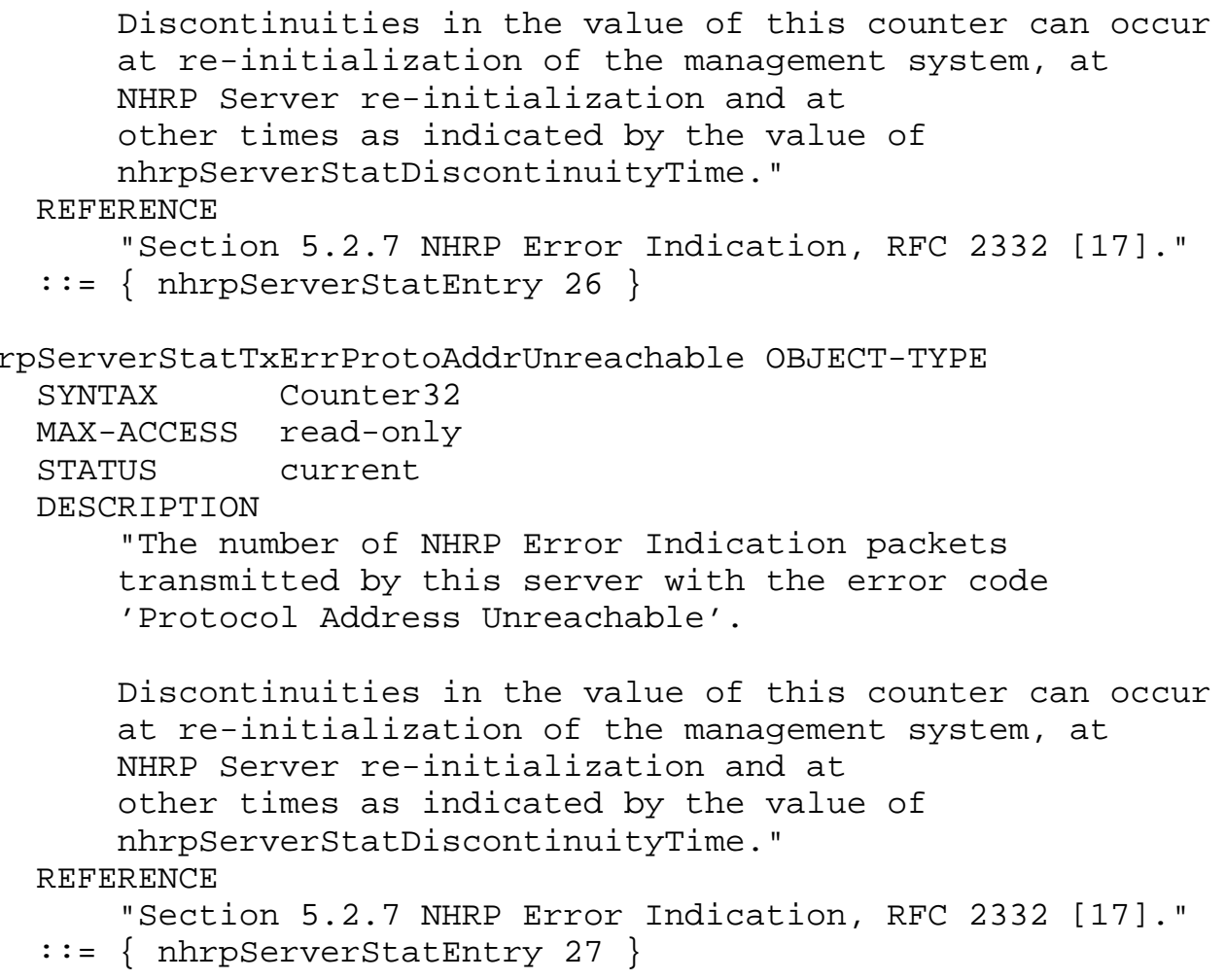




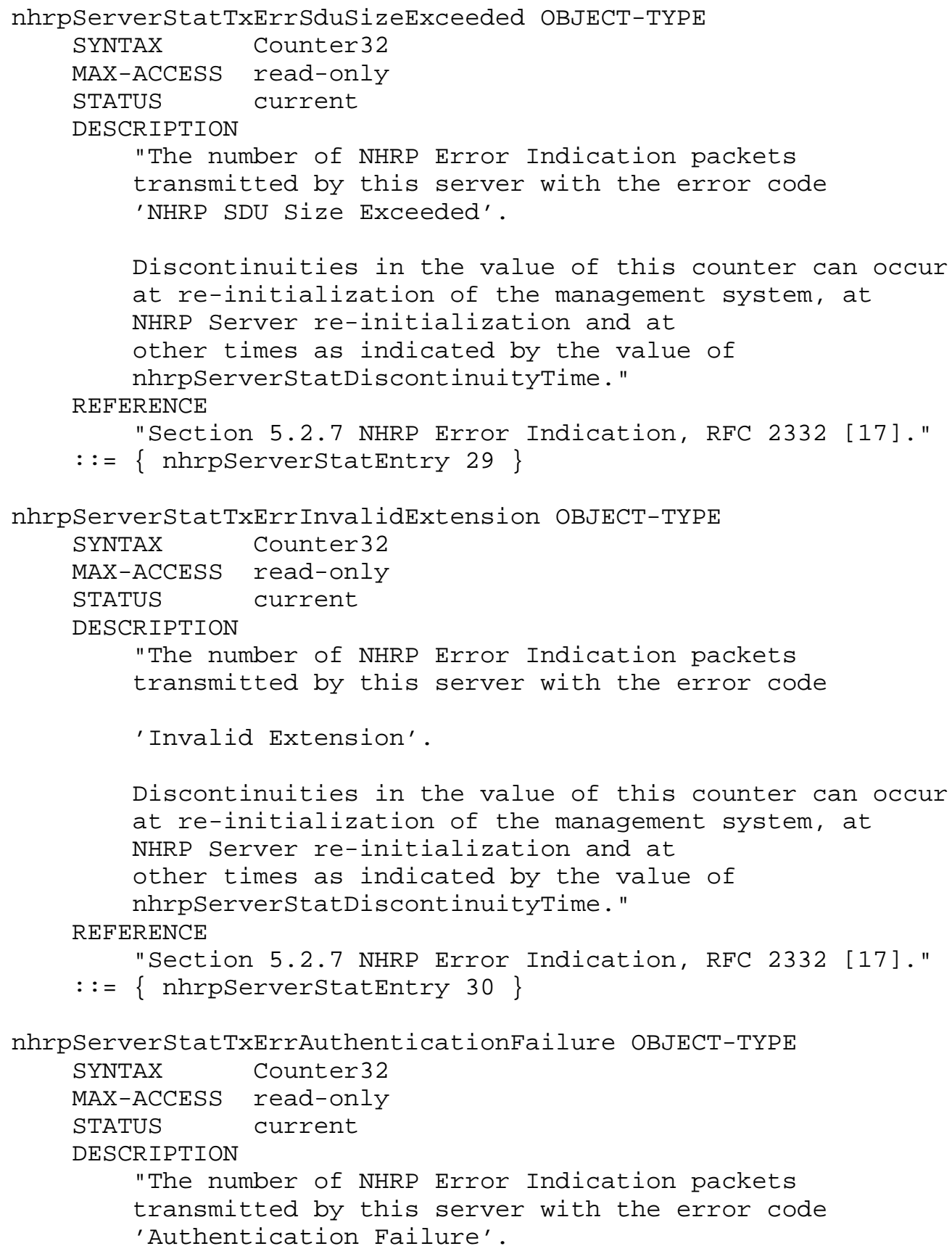




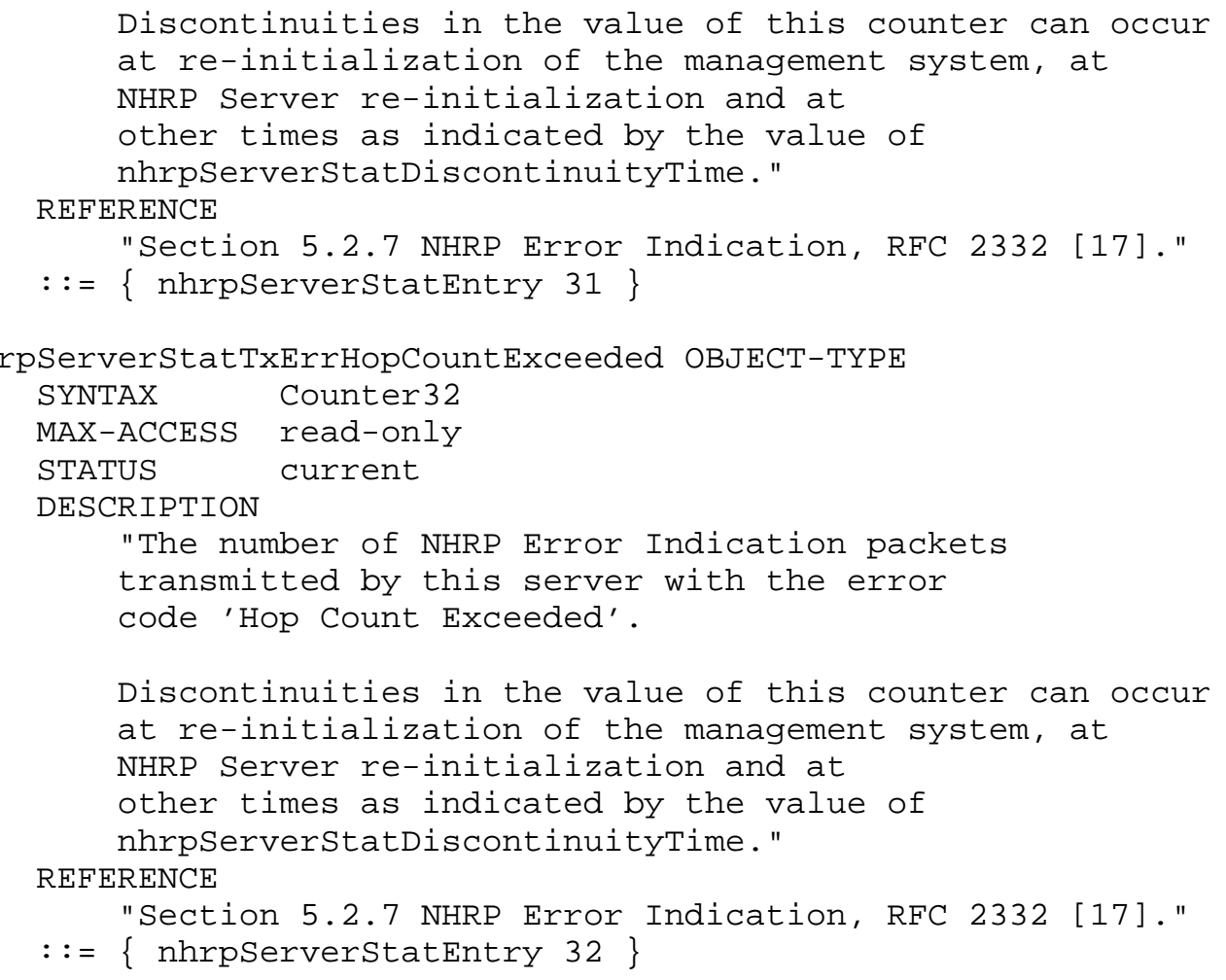

nhrpServerStatFwResolveReq OBJECT-TYPE

$\begin{array}{ll}\text { SYNTAX } & \text { Counter32 } \\ \text { MAX-ACCESS } & \text { read-only } \\ \text { STATUS } & \text { current }\end{array}$

DESCRIPTION

"The number of NHRP Resolution Requests

forwarded by this server acting as a transit NHS.

Discontinuities in the value of this counter can occur at re-initialization of the management system, at NHRP Server re-initialization and at 
DESCRIPTION

"The number of NHRP Resolution Replies forwarded

by this server acting as a transit NHS.

Discontinuities in the value of this counter can occur at re-initialization of the management system, at NHRP Server re-initialization and at other times as indicated by the value of nhrpserverstatDiscontinuityTime."

$::=\{$ nhrpserverstatEntry 34$\}$

nhrpServerStatFwRegisterReq OBJECT-TYPE

SYNTAX Counter32

MAX-ACCESS read-only

STATUS current

DESCRIPTION

"The number of NHRP Registration Requests forwarded by this server acting as a transit NHS.

Discontinuities in the value of this counter can occur at re-initialization of the management system, at NHRP Server re-initialization and at other times as indicated by the value of nhrpserverstatDiscontinuityTime."

$::=\{$ nhrpserverstatentry 35$\}$

nhrpServerStatFwRegisterReply OBJECT-TYPE

SYNTAX Counter32

MAX-ACCESS read-only

STATUS current

DESCRIPTION

"The number of NHRP Registration Replies forwarded

by this server acting as a transit NHS.

Discontinuities in the value of this counter can occur at re-initialization of the management system, at NHRP Server re-initialization and at other times as indicated by the value of nhrpserverstatDiscontinuityTime."

$::=\{$ nhrpserverStatEntry 36$\}$

nhrpServerStatFwPurgeReq OBJECT-TYPE

SYNTAX Counter32

MAX-ACCESS read-only

STATUS current

DESCRIPTION

"The number of NHRP Purge Requests forwarded by this server acting as a transit NHS. 


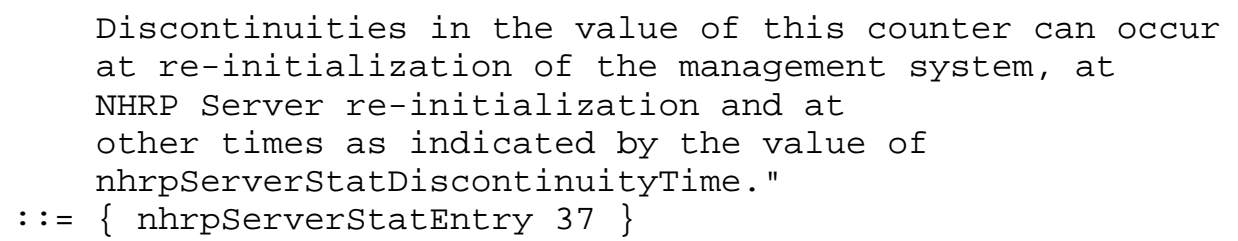


local management subsystem or the NHRP Server re-initialization associated with this entry, then this object contains a zero value." REFERENCE

"RFC 2233 [18]."

$::=\{$ nhrpserverstatentry 40$\}$

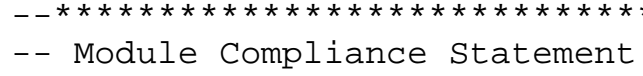

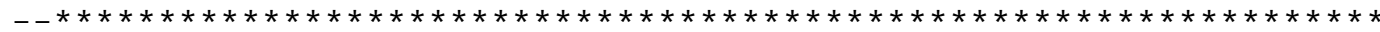

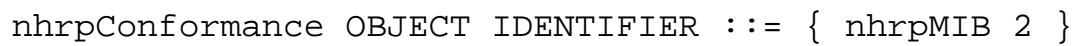

nhrpcompliances

OBJECT IDENTIFIER $::=\{$ nhrpConformance 1$\}$

nhrpGroups

OBJECT IDENTIFIER : := $\{$ nhrpConformance 2$\}$

nhrpModuleCompliance MODULE-COMPLIANCE

STATUS current

DESCRIPTION

"The compliance statement for the NHRP MIB." MODULE -- this module

MANDATORY-GROUPS \{nhrpGeneralGroup \}

GROUP nhrpClientGroup

DESCRIPTION

"This group must be supported only by stations that are NHRP clients."

GROUP nhrpserverGroup

DESCRIPTION

"This group must be supported only by stations that are NHRP servers."

$::=\{$ nhrpcompliances 1$\}$

nhrpGeneralGroup OBJECT-GROUP

OBJECTS \{

nhrpNext Index,

nhrpCachePrefixLength, nhrpCacheNext HopInternetworkAddr,

nhrpCacheNbmaAddrType,

nhrpCacheNbmaAddr,

nhrpCacheNbmaSubaddr, nhrpCacheType,

nhrpcachestate, 


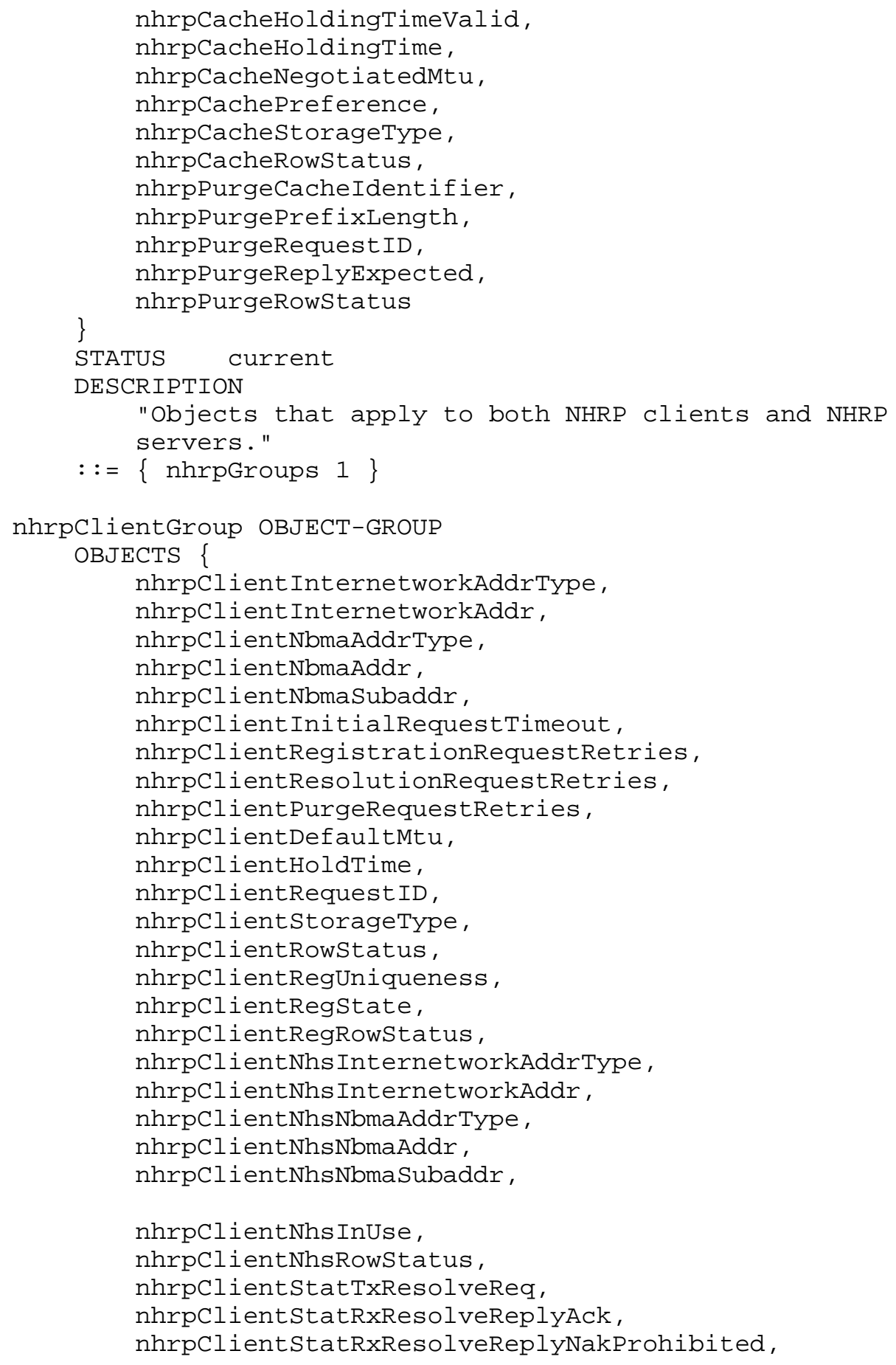




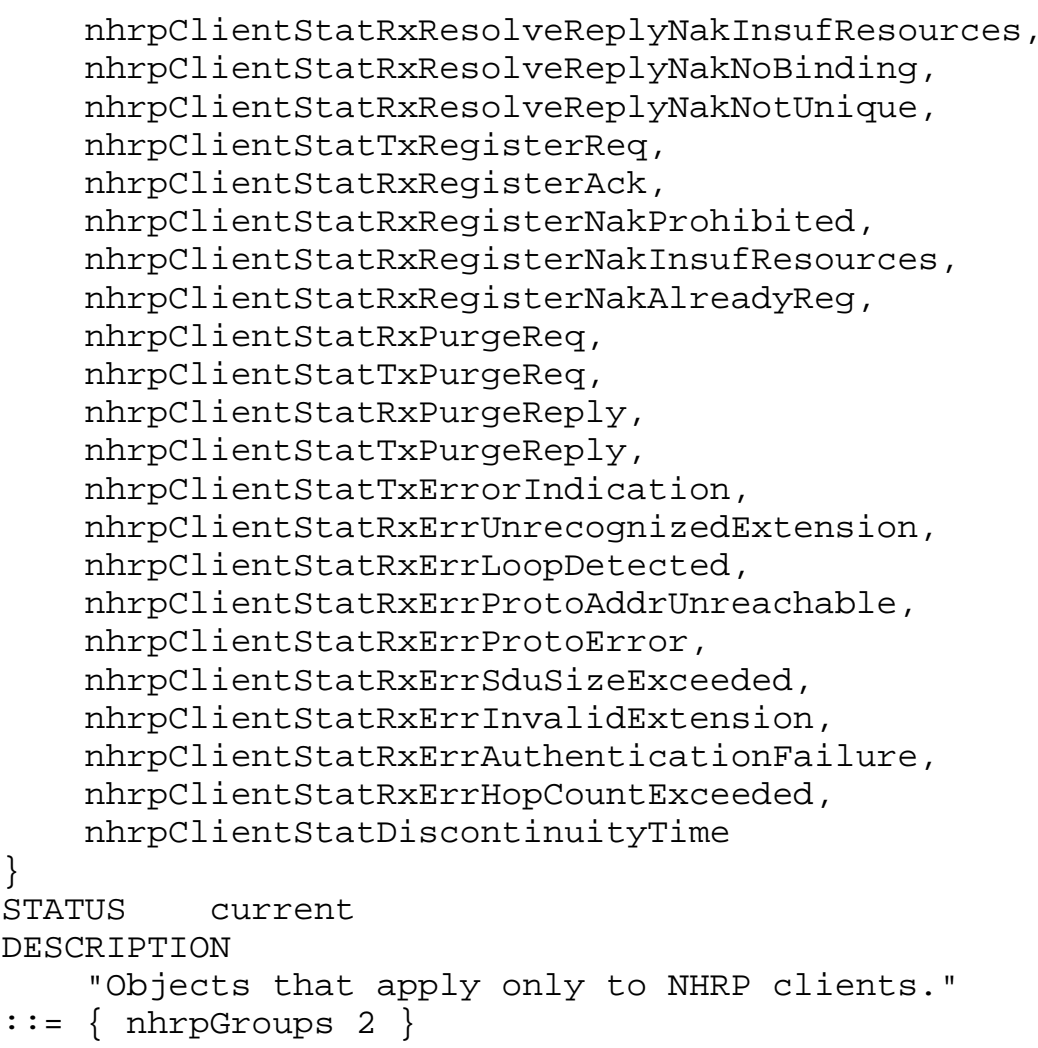




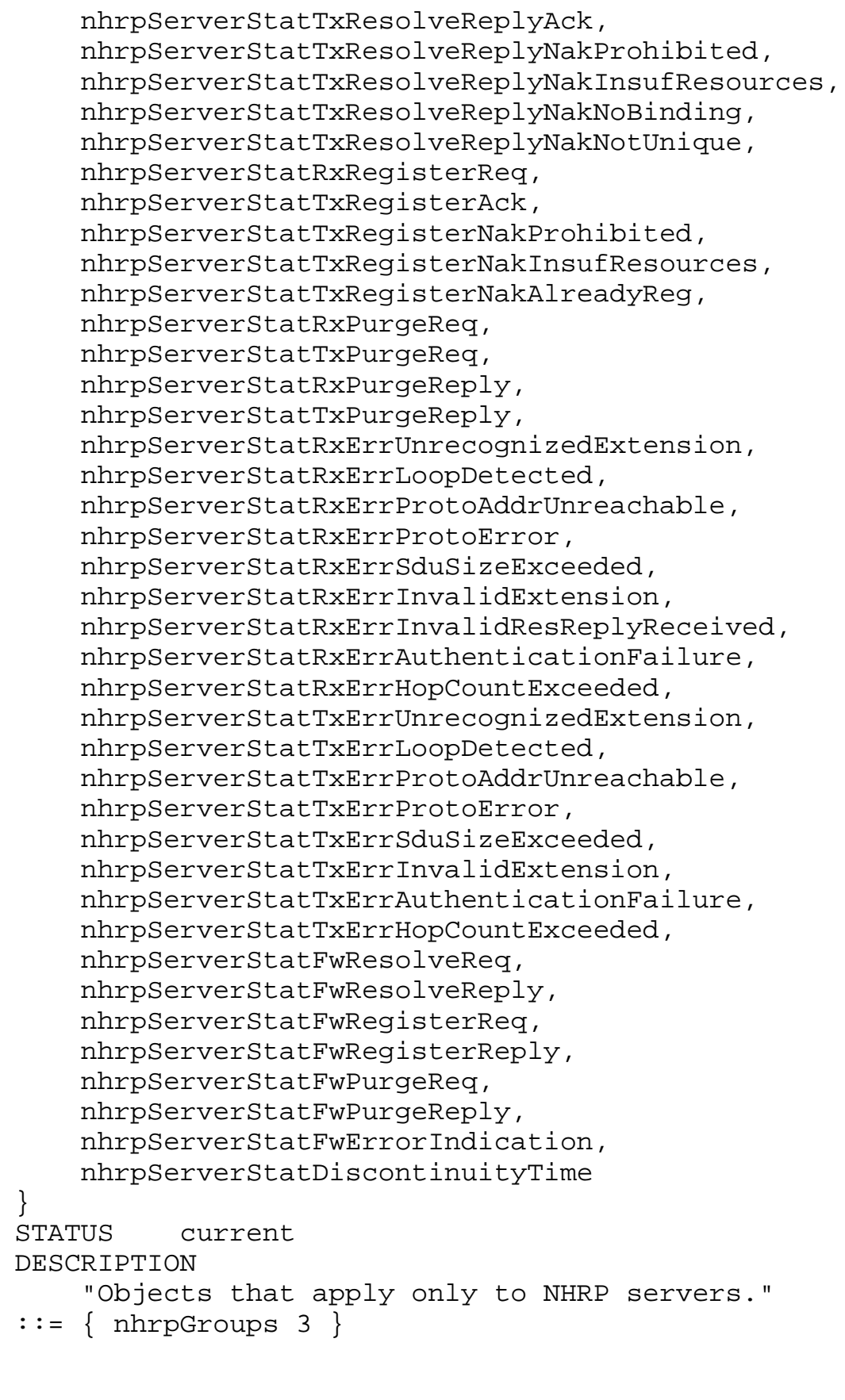

END 


\section{IANA Considerations}

The Internet Assigned Numbers Authority (IANA) has been and continues to be responsible for maintaining the ADDRESS FAMILY NUMBERS

(http://www.isi.edu/in-notes/iana/assignments/address-family-numbers) name space assignments. The IANA has placed this list in a MIB module, such that it may be imported into other MIBs. The motivation for doing this is to allow MIBs to not have to change when a new assignment is made to the ADDRESS FAMILY NUMBERS. This is very similar to the motivation behind the IANAifType-MIB.

Any additions or changes to the list of ADDRESS FAMILY NUMBERS registered via IANA will be done as they have in the past and this document does not propose any changes to the ADDRESS FAMILY NUMBERS other than to place them into a MIB, which can be found via anonymous FTP at: ftp://ftp.isi.edu/mib/ianaaddressfamilynumbers.mib.

\section{Security}

There are a number of management objects defined in this MIB that have a MAX-ACCESS clause of read-write and/or read-create. Such objects may be considered sensitive or vulnerable in some network environments. The support for SET operations in a non-secure environment without proper protection can have a negative effect on network operations.

The NHRP Protocol, RFC 2332 [17], Section 5.2.4.4 discusses security. There is an authentication option which should be utilized to authenticate the source and also provide data integrity to the NHRP payload. This MIB does not contain any managed objects which configure or expose security information such as that needed for NHRP authentication or data integrity.

The following items were deemed to jeopardize security and thus, were NOT added to this MIB. Items denoted as (configurable) are those which would need values. Items denoted as (read-only) are those which would provide information. Although the NHRP Protocol [17], requires or has this information, exposing it in a MIB would jeopardize the entire NBMA domain where NHRP was being used. Therefore, these items have been omitted from the MIB. 
1. (configurable) enable/disable security

2. (configurable) SPI (security parameter index). Depending upon the implementation,

there may be multiple SPIs, and these would be configurable also. For example, if the implementation switched to a different SPI after a given time.

3. (configurable) algorithm. The HMAC-MD5-128 is the default hash algorithm.

4. (configurable) lifetime value in seconds.

5. (read-only) key.

6. (read-only) list of users who have access to the above information.

7. Intellectual Property

The IETF takes no position regarding the validity or scope of any intellectual property or other rights that might be claimed to pertain to the implementation or use of the technology described in this document or the extent to which any license under such rights might or might not be available; neither does it represent that it has made any effort to identify any such rights. Information on the IETF's procedures with respect to rights in standards-track and standards-related documentation can be found in BCP-11. Copies of claims of rights made available for publication and any assurances of licenses to be made available, or the result of an attempt made to obtain a general license or permission for the use of such proprietary rights by implementors or users of this specification can be obtained from the IETF secretariat.

The IETF invites any interested party to bring to its attention any copyrights, patents or patent applications, or other proprietary rights which may cover technology that may be required to practice this standard. Please address the information to the IETF Executive Director.

\section{Acknowledgments}

This document is a product of the IETF's Internetworking Over NBMA Networks (ion) Working Group.

The authors would like to thank Avri Doria (Bytex) for the first draft of the NHRP MIB and Keith McCloghrie (cisco) and David Horton (CITR) for their feedback and suggestions. Also, we would like to thank Naganand Doraswamy (Bay Networks) for assistance with the "Security Considerations" section. 


\section{References}

[1] Harrington, D., Presuhn, R. and B. Wijnen, "An Architecture for Describing SNMP Management Frameworks", RFC 2571, April 1999.

[2] Rose, M. and K. McCloghrie, "Structure and Identification of Management Information for TCP/IP-based Internets", STD 16, RFC 1155, May 1990 .

[3] Rose, M. and K. McCloghrie, "Concise MIB Definitions", STD 16, RFC 1212, March 1991.

[4] Rose, M., "A Convention for Defining Traps for use with the SNMP", RFC 1215, March 1991.

[5] McCloghrie, K., Perkins, D., Schoenwaelder, J., Case, J., Rose, M. and S. Waldbusser, "Structure of Management Information Version 2 (SMIV2)", STD 58, RFC 2578, April 1999.

[6] McCloghrie, K., Perkins, D., Schoenwaelder, J., Case, J., Rose, M. and S. Waldbusser, "Textual Conventions for SMIv2", STD 58, RFC 2579, April 1999.

[7] McCloghrie, K., Perkins, D., Schoenwaelder, J., Case, J., Rose, M. and S. Waldbusser, "Conformance Statements for SMIv2", STD 58, RFC 2580, April 1999.

[8] Case, J., Fedor, M., Schoffstall, M. and J. Davin, "Simple Network Management Protocol", STD 15, RFC 1157, May 1990.

[9] Case, J., McCloghrie, K., Rose, M. and S. Waldbusser, "Introduction to Community-based SNMPv2", RFC 1901, January 1996.

[10] Case, J., McCloghrie, K., Rose, M. and S. Waldbusser, "Transport Mappings for Version 2 of the Simple Network Management Protocol (SNMPv2) ", RFC 1906, January 1996.

[11] Case, J., Harrington D., Presuhn R. and B. Wijnen, "Message Processing and Dispatching for the Simple Network Management Protocol (SNMP)", RFC 2572, April 1999.

[12] Blumenthal, U. and B. Wijnen, "User-based Security Model (USM) for version 3 of the Simple Network Management Protocol (SNMPv3)", RFC 2574, April 1999. 
[13] Case, J., McCloghrie, K., Rose, M. and S. Waldbusser, "Protocol Operations for Version 2 of the Simple Network Management Protocol (SNMPv2)", RFC 1905, January 1996.

[14] Levi, D., Meyer, P. and B. Stewart, "SNMPv3 Applications", RFC 2573, April 1999.

[15] Wijnen, B., Presuhn, R. and K. McCloghrie, "View-based Access Control Model (VACM) for the Simple Network Management Protocol (SNMP) ", RFC 2575, April 1999.

[16] Case, J., Mundy, R., Partain, D. and B. Stewart, "Introduction to Version 3 of the Internet-standard Network Management Framework", RFC 2570, April 1999.

[17] Luciani, J. V., Katz, D., Piscitello, D. and B. Cole, "NBMA Next Hop Resolution Protocol (NHRP)", RFC 2332, December 1997.

[18] McCloghrie, K. and F. Kastenholz, "The Interfaces Group MIB using SMIv2", RFC 2233, November 1997.

[19] Tesink, K., Editor, "Definitions of Managed Objects for ATM Management", RFC 2515, February 1999.

[20] Narten, T. and H. Alvestrand, "Guidelines for Writing an IANA Considerations section in RFCs", BCP 26, RFC 2434, October 1998.

[21] Bradner, S., "Key words for use in RFCs to Indicate Requirement Levels", BCP 14, RFC 2119, March 1997.

[22] Bradner, S., "The Internet Standards Process -- Revision 3", BCP 9, RFC 2026, October 1996.

[23] Cucchiara, J., editor, "Multiprotocol Over ATM Version 1.0 MIB", af-mpoa-0092.000, ATM Forum, July 1998.

[24] Fredette, A., editor, "Multiprotocol Over ATM Version 1.0", af-mpoa-0087.000, ATM Forum, May 1997.

[25] Laubach, M., and J. Halpern, "Classical IP and ARP over ATM", RFC 2225, April 1998.

[26] Greene, M., J. Luciani, K. White and T. Kuo, "Definitions of Managed Objects for Classical IP and ARP Over ATM Using SMIv2", RFC 2320, April 1998. 
10. Authors' Addresses

James V. Luciani

Bay Networks

3 Federal Street

Mail Stop: BL3-03

Billerica, MA 01821

Phone: (978) 288-4734

EMail: luciani@baynetworks.com

Maria Greene

Contractor

Xedia, Corp.

119 Russell Dr.

Littleton, MA 01460

EMail: maria@xedia.com

Joan Cucchiara

IronBridge Networks

55 Hayden Ave.

Lexington, MA 02421

Phone: (781) 372-8236

EMail: joan@ironbridgenetworks.com 
12. Full Copyright statement

Copyright (C) The Internet Society (1999). All Rights Reserved.

This document and translations of it may be copied and furnished to others, and derivative works that comment on or otherwise explain it or assist in its implementation may be prepared, copied, published and distributed, in whole or in part, without restriction of any kind, provided that the above copyright notice and this paragraph are included on all such copies and derivative works. However, this document itself may not be modified in any way, such as by removing the copyright notice or references to the Internet society or other Internet organizations, except as needed for the purpose of developing Internet standards in which case the procedures for copyrights defined in the Internet Standards process must be followed, or as required to translate it into languages other than English.

The limited permissions granted above are perpetual and will not be revoked by the Internet society or its successors or assigns.

This document and the information contained herein is provided on an "AS IS" basis and THE INTERNET SOCIETY AND THE INTERNET ENGINEERING TASK FORCE DISCLAIMS ALL WARRANTIES, EXPRESS OR IMPLIED, INCLUDING BUT NOT LIMITED TO ANY WARRANTY THAT THE USE OF THE INFORMATION HEREIN WILL NOT INFRINGE ANY RIGHTS OR ANY IMPLIED WARRANTIES OF MERCHANTABILITY OR FITNESS FOR A PARTICULAR PURPOSE.

Acknowledgement

Funding for the RFC Editor function is currently provided by the Internet society. 